\title{
Tiger beetles (Coleoptera, Cicindelidae) of Northern Mindanao region (Philippines): checklist, distributional maps, and habitats
}

\author{
Dale Ann P. Acal', Jürgen Wiesner², Olga M. Nuñeza', Radomir Jaskuła ${ }^{3}$ \\ I Department of Biological Sciences, College of Science and Mathematics, Mindanao State University-Iligan \\ Institute of Technology, Andres Bonifacio Ave., Tibanga, Iligan City 9200, Philippines 2 Dresdener Ring 11, \\ D-38444, Wolfsburg, Germany 3 Department of Invertebrate Zoology and Hydrobiology, Faculty of Biology \\ and Environmental Protection, University of Lodz, Banacha 12/16, 90-237, Eódź, Poland \\ Corresponding author: Radomir Jaskuła (radomir.jaskula@biol.uni.lodz.pl)
}

Academic editor: R. Vermeulen | Received 13 March 2019 | Accepted 12 January 2021 | Published 12 February 2021

http://zoobank.org/390FEA39-DEBA-4406-B99F-BC6625821960

Citation: Acal DAP, Wiesner J, Nuńeza OM, Jaskuła R (2021) Tiger beetles (Coleoptera, Cicindelidae) of Northern Mindanao region (Philippines): checklist, distributional maps, and habitats. ZooKeys 1017: 37-75. https://doi. org/10.3897/zookeys.1017.34500

\begin{abstract}
The knowledge about tiger beetle fauna of the Northern Mindanao region (Philippines) is summarized based on literature data and new records. Thirty species classified in ten genera (Tricondyla, Neocollyris, Protocollyris, Therates, Prothyma, Heptodonta, Thopeutica, Lophyra, Calomera, and Cylindera) were documented from the area (56\% of tiger beetle fauna of Mindanao and $21 \%$ of Philippine species). Twelve species were noted from Northern Mindanao region for the first time, including five taxa, Neocollyris speciosa, Calomera angulata, Cylindera minuta, Lophyra striolata tenuiscripta, and Thopeutica virginea, not recorded from Mindanao before. Distribution maps for all recorded species and the first photographs of habitats for some species in Mindanao and/or in the Philippines are provided. Eight species (27\% of recorded fauna) were noted from riverine habitats while 18 tiger beetles (60\%) were typical forest taxa; in the case of four species, their habitats in Northern Mindanao region are not known.
\end{abstract}

\section{Keywords}

Endemic species, Calomera, Cylindera, diversity, distribution, Heptodonta, identification key, Lophyra, Neocollyris, Protocollyris, Prothyma, Therates, Tricondyla

Copyright Dale Ann P.Acal et al. This is an open access article distributed under the terms of the Creative Commons Attribution License (CC BY 4.0), which permits unrestricted use, distribution, and reproduction in any medium, provided the original author and source are credited. 


\section{Introduction}

Tiger beetles (Coleoptera: Cicindelidae) are a beetle family (López-López and Vogler 2017; Duran and Gough 2020) of more than 2850 species distributed world-wide, but with the larger number of taxa occurring in tropical regions (Cassola and Pearson 2000; Wiesner 2020). With 162 taxa (including 144 species) actually known from the country the tiger beetle fauna of the Philippines is recognized as one of the most diverse in the world (Cabras et al. 2016a; Dheurle 2016, 2019; Zettel and Pangantihon 2017; Zettel and Wiesner 2018; Anichtchenko and Medina 2019, 2020; Medina et al. 2019, 2020; Görn 2020). Moreover, it can be characterized by high percentage of endemic species as more than $85 \%$ of Cicindelidae are noted only from this country (Cassola and Pearson 2000; Cabras et al. 2016a; Dheurle 2016, 2019; Zettel and Pangantihon 2017; Zettel and Wiesner 2018; Anichtchenko and Medina 2019, 2020; Medina et al. 2019, 2020c; Görn 2020), with particular species often noted only on single islands (Cabras et al. 2016a). It can be expected that such high diversity values results from both geographical location of the country in the tropical region as well as the occurrence of a large number of geographically isolated islands that influence the evolution of endemic species.

Based on previous studies, ten genera and 54 species of tiger beetles were recorded on Mindanao Island: Tricondyla Latreille, 1822 (7 species), Protocollyris Mandl, 1975 (3 species), Neocollyris Horn, 1901 (12 species), Therates Latreille, 1817 (4 species), Prothyma Hope, 1838 (6 species), Heptodonta Hope, 1838 (2 species), Calomera Motschulsky, 1862 (3 species), Lophyra Motschulsky, 1859 (1 species), Thopeutica Chaudoir, 1861 (8 species), and Cylindera Westwood, 1831 (4 species) (Wiesner 1992; Cassola 2000, 2011; Naviaux 1994, 2002; Cassola and Ward 2004; Dheurle 2015, 2019; Cabras et al. 2016a; Anichtchenko and Medina 2019; Görn 2020; Medina et al. 2019, 2020c).

Although more than 50 tiger beetle species are known as occurring on Mindanao Island (36\% of Philippine fauna), for many of these taxa only single records are known (Cabras et al. 2016a). Hence, little is known about the general distribution of many species and from many regions no data are available. As Philippine tiger beetle fauna includes many endemic taxa, lack of distributional data often do not allow estimates of species ranges, abundance, or habitat selection and as a consequence, also their threats. As degradation of wildlife both in the Philippines and Mindanao is significant due to different human activities (e.g., deforestation for agricultural land, human pressures because of overpopulation; Lasco et al. 2008; Navarrete et al. 2018), even simple faunistic data may play an important role in the conservation of this beetle group.

In the present paper we focus on the region of Northern Mindanao where no regular studies on tiger beetle species were previously done. As a result, the first checklist of Cicindelidae occurring in this region as well as distributional maps for all known species occurring in the area, and photographs of habitats for the 12 recorded taxa are provided. This paper may serve as a baseline for further studies on this beetle family not only in the Northern Mindanao but also in other regions in the country. 


\section{Materials and methods}

Northern Mindanao geographically lies within latitude $7^{\circ} 15^{\prime}$ to $9^{\circ} 15^{\prime} \mathrm{N}$ and longitude $123^{\circ} 30^{\prime}$ to $125^{\circ} 30^{\prime} \mathrm{E}$, is bound on the north by the Bohol Sea, on the west by Zamboanga provinces, on the east by Agusan and Davao provinces, on the south by Lanao del Sur and Cotabato. The whole region covers a total land area of $19,279.60 \mathrm{~km}^{2}$ and more than $60 \%$ of the region's area are classified as forest land. Geologically, this region is formed of a combination of coastal areas, rivers, falls, volcanoes, highlands with flat terrain, rugged and faulted mountains with rich soil, abundant minerals, and agricultural resources. Since the region is located outside the typhoon belt area, rainfall is evenly distributed throughout the year (Dejarme-Calalang and Colinet 2014; Bouquet 2017). Northern Mindanao comprises five provinces: Bukidnon, Camiguin, Lanao del Norte, Misamis Occidental, and Misamis Oriental (Figure 1). Regular studies were done in sixteen sampling sites in the Northern Mindanao region, including riverine areas, secondary forests, and coastal areas (Table 1, Figures 2-4), and most of the tiger beetle material used in this study was collected using entomological hand nets during field work in 2017-2019. Some additional data were provided from earlier studies, including published data (Wiesner 1988; Naviaux 1994, 2002; Cassola 2000, 2011; Dheurle 2015, 2017; Cabras et al. 2016a; Görn 2020).

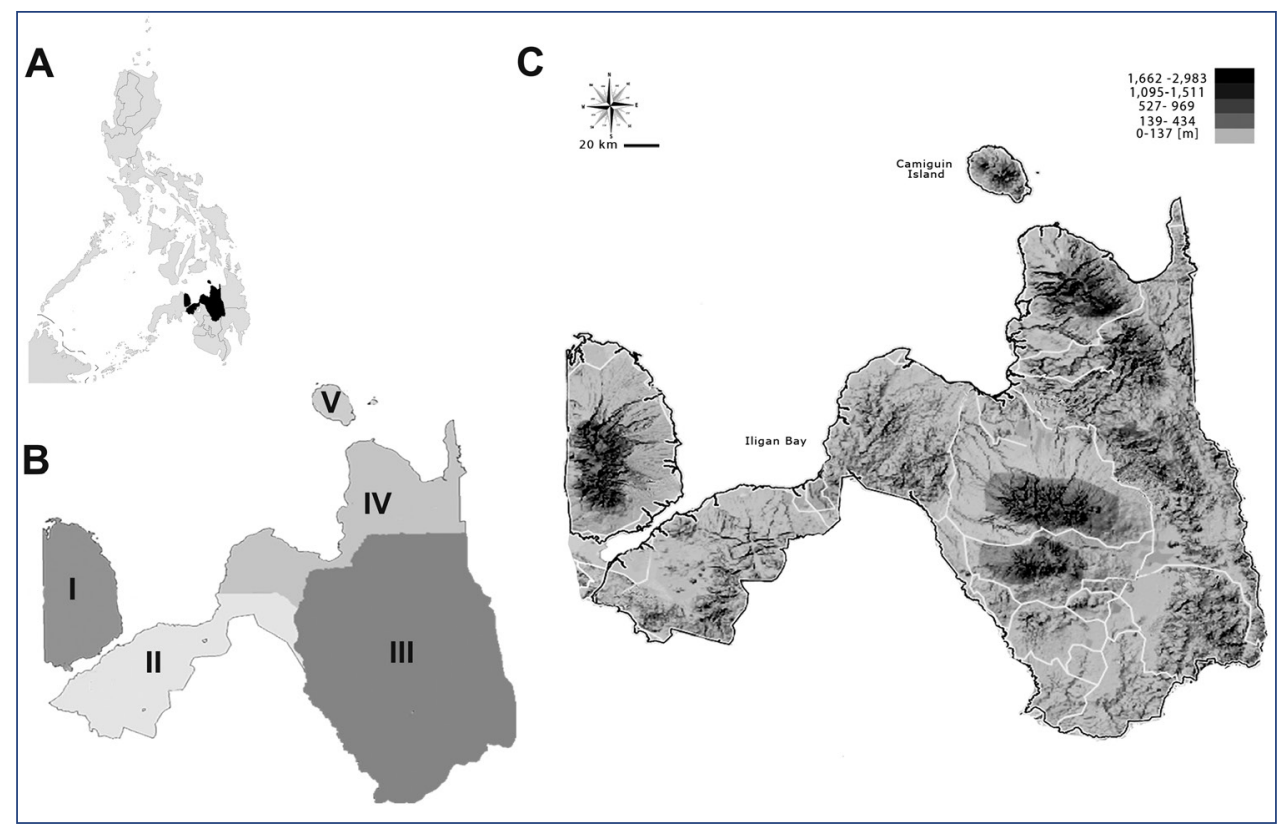

Figure I. Map of Northern Mindanao region $\mathbf{A}$ in relation to entire Mindanao island and the remaining Philippine archipelago B administrative provinces (I - Misamis Occidental, II - Lanao del Norte, III - Bukidnon, IV - Misamis Oriental, V - Camiguin) C detailed physical map. 
Table I. Sampling sites in Northern Mindanao region were regular studies were done in years 2017 and/or 2018.

\begin{tabular}{|c|c|c|}
\hline Site & Sampling site & GPS coordinates \\
\hline 1. & Barangay Bulokbulok, Municipality of Mambajao, Camiguin Island, Camiguin Province & $9^{\circ} 15^{\prime} 9^{\prime \prime} \mathrm{N}, 124^{\circ} 42^{\prime} 31^{\prime \prime E}$ \\
\hline 2. & Looc River, Barangay Mainit, Municipality of Catarman, Camiguin Island, Camiguin Province & $9^{\circ} 10^{\prime} 30^{\prime \prime} \mathrm{N}, 124^{\circ} 40^{\prime} 44^{\prime \prime} \mathrm{E}$ \\
\hline 3. & Sagay River, Barangay Bonbon, Municipality of Sagay, Camiguin Island, Camiguin Province & $9^{\circ} 6^{\prime} 18^{\prime \prime} \mathrm{N}, 124^{\circ} 43^{\prime} 57^{\prime \prime} \mathrm{E}$ \\
\hline 4. & Barangay Bura, Municipality of Catarman, Camiguin Island, Camiguin Province & $9^{\circ} 10^{\prime} 4.7^{\prime \prime} \mathrm{N}, \mathrm{E} 124^{\circ} 39^{\prime} 23^{\prime \prime} \mathrm{E}$ \\
\hline 5. & Barangay Poblacion, Municipality of Mambajao, Camiguin Island, Camiguin Province & $9^{\circ} 13^{\prime} 24^{\prime \prime N}, 124^{\circ} 41^{\prime} 47^{\prime \prime E}$ \\
\hline 6. & Barangay Umagus, Municipality of Lagonglong, Misamis Oriental Province & $8^{\circ} 48^{\prime} 11^{\prime \prime} \mathrm{N}, 124^{\circ} 48^{\prime} 53^{\prime \prime} \mathrm{E}$ \\
\hline 7. & Cabulaway River, Municipality of Balingasag, Misamis Oriental Province & $8^{\circ} 46^{\prime} 9^{\prime \prime} \mathrm{N}, 124^{\circ} 48^{\prime} 2^{\prime \prime} \mathrm{E}$ \\
\hline 8. & Barangay Kalasungay, Malaybalay City, Bukidnon Province & $8^{\circ} 11^{\prime} 28^{\prime \prime} \mathrm{N}, 125^{\circ} 5^{\prime} 54^{\prime \prime} \mathrm{E}$ \\
\hline 9. & Barangay Can-ayan, Malaybalay City, Bukidnon Province & $8^{\circ} 11^{\prime} 31^{\prime \prime N}, 125^{\circ} 9^{\prime} 13^{\prime \prime E}$ \\
\hline 10. & Barangay Bonbonon, Iligan City, Lanao del Norte Province & $8^{\circ} 15^{\prime} 56^{\prime \prime} \mathrm{N}, 124^{\circ} 18^{\prime} 37^{\prime \prime} \mathrm{E}$ \\
\hline 11. & Tubod River, Barangay Merilla, Iligan City, Lanao del Norte Province & $8^{\circ} 12^{\prime} 17^{\prime \prime} \mathrm{N}, 124^{\circ} 15^{\prime} 24^{\prime \prime} \mathrm{E}$ \\
\hline 12. & Barangay Esperanza, Municipality of Bacolod, Lanao del Norte Province & $8^{\circ} 10^{\prime} 12^{\prime \prime} \mathrm{N}, 124^{\circ} 0^{\prime} 22^{\prime \prime} \mathrm{E}$ \\
\hline 13. & Barangay Mati, Municipality of Bacolod, Lanao del Norte Province & $8^{\circ} 9^{\prime} 4^{\prime \prime N}, 124^{\circ} 0^{\prime} 57^{\prime \prime} \mathrm{E}$ \\
\hline 14. & Barangay San Isidro, Municipality of Sinacaban, Misamis Occidental Province & $8^{\circ} 17^{\prime} 5^{\prime \prime N}, 123^{\circ} 47^{\prime} 5^{\prime \prime E}$ \\
\hline 15. & Barangay San Lorenzo, Municipality of Sinacaban, Misamis Occidental Province & $8^{\circ} 17^{\prime} 10^{\prime \prime} \mathrm{N}, 123^{\circ} 41^{\prime} 43 " \mathrm{E}$ \\
\hline 16. & Mt. Agad-Agad, Iligan City, Lanao del Norte Province & $8^{\circ} 12^{\prime} 49.34 " \mathrm{~N}, 124^{\circ} 16^{\prime} 9.66^{\prime \prime} \mathrm{E}$ \\
\hline 17. & Mimbilisan Protected Landscape, Misamis Oriental Province & $8.94884 \mathrm{~N}, 124.86517 \mathrm{E}$ \\
\hline 18. & Municipality of Lopez Jaena, Misamis Occidental Province & $8^{\circ} 33^{\prime} 00^{\prime \prime} \mathrm{N}, 123^{\circ} 46^{\prime} 00^{\prime \prime} \mathrm{E}$ \\
\hline
\end{tabular}

Material is currently deposited in the authors' collections:

DAC D.A.P. Acal Collection (Illigan City, Philippines);

JWC J. Wiesner Collection (Wolfsburg, Germany);

RJC R. Jaskuła Collection (Łódź, Poland).

\section{Checklist of tiger beetles of Northern Mindanao}

Family Cicindelidae Latreille, 1802

Subfamily Cicindelinae Latreille, 1802

Tribus Collyridini Brullé, 1834

Subtribus Tricondylina Naviaux, 1991

Genus Tricondyla Latreille, 1822

The genus Tricondyla is represented in the Philippine islands by 14 species, including seven recorded from Mindanao Island (Naviaux 2002; Cabras et al. 2016a).

\section{Subgenus Tricondyla Latreille, 1822}

\section{Tricondyla (Tricondyla) aptera punctipennis Chevrolat, 1841}

Figures 4B, 5A, 6A

General distribution. Subspecies known from Indonesia and Philippines. In the Philippines it was found in Sibuyan, Samar, Cebu, and Mindanao; in Mindanao Island it was recorded only in the Northern Mindanao region (Cabras et al. 2016a). 
Literature data for Northern Mindanao. Bukidnon province: Impasung-ong (Cabras et al. 2016a).

Material examined. Bukidnon Province: Mt. Kitanglad, 28.VII.1990, $1 \delta^{\Uparrow} 1$, ex coll. Y. Nishiyama (JWC); [no detailed locality], 1977, 10 , leg. R. Lumawig (JWC); Lanao del Norte province: Mt. Agad-agad, $8^{\circ} 12^{\prime} 49.34 " \mathrm{~N}, 124^{\circ} 16^{\prime} 9.66^{\prime \prime} \mathrm{E}, 470 \mathrm{~m}$ a.s.l., 19.11.2018, 1ठ, leg. R. Jaskuła et D.A.P. Acal (RJC).

Habitat. Forest species found on vertical and fallen tree trunks, sometimes in forest floor; occasionally also outside the forest (but close to the trees).

Remarks. Larva of nominal subspecies was described by Trautner and Schawaller (1996), who observed it hunting during night period in the bark of tree (e.g., Samanea saman).

\section{Tricondyla (Tricondyla) elongata Horn, 1906}

Figure 6B

General distribution. Species endemic to the Philippines, where it was recorded from Luzon, Visayas, and Mindanao; in Mindanao recorded only in Northern Mindanao, Davao, and Bangsamoro Autonomous Region in Muslim Mindanao regions (Cabras et al. 2016a, b; Marohomsalic et al. 2021).

Literature data for Northern Mindanao. Bukidnon province: Lantapan and Impasung-ong (Cabras et al. 2016a); Misamis Occidental province: Mt. Malindang (Cabras et al. 2016a); Misamis Oriental province: Mt. Balatucan-Lumot (Cabras et al. 2016a).

Material examined. Bukidnon province: Mt. Kitanglad, 28.VII.1990, $1 \delta 2$ 万우,

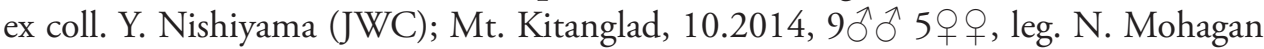
(JWC); Lanao del Norte province: Mount Agad-agad, 8¹2'49.34"N, 124¹6'9.66"E, ca. 470 m a.s.l., 11.2019, 1 ex., leg. J. Ebina, M. L. Lumontod, G. C. Café (RJC); Dodiongan Falls, Iligan City - Barangay Bonbonon, 8.271457N, 124.314140E, 47 m a.s.l., 11.2019, 1 ex., leg. R. Jaskuła (RJC); Tinago Falls, Iligan City - Barangay Ditucalan, 8.159820N, 124.185460E, 11.2019, 1 ex., leg. Ł. Trębicki (RJC); Misamis Oriental province: Cagayan de Oro, Malasag forest, 23.05.1978, 1q, leg. A. Bandinelli (JWC).

Habitat. Forest, arboreal species found on vertical and fallen tree trunks. Marohomsalic et al. (2021) recorded native and invasive tree species having extrafloral nectaries as favorite hunting areas for this species in the human-disturbed habitats.

\section{Tricondyla (Tricondyla) gracilis Naviaux, 2002}

Figure 6C

General distribution. Species endemic to Philippines where it was found only in Mindanao and Romblon islands (Naviaux 2002; Cabras et al. 2016a); in Mindanao known from Davao and Northern Mindanao regions (Naviaux 2002). 
Literature data for Northern Mindanao. Misamis Oriental province: Malasag forest Cagayan de Oro (Naviaux 2002).

Habitat. Forest, arboreal species found on vertical and fallen tree trunks.

Subgenus Stenotricondyla Naviaux, 2002

Tricondyla (Stenotricondyla) cyanipes Eschscholtz, 1829

Figure 6D

General distribution. Species endemic to the Philippines where it was found in Luzon, Leyte, Sibuyan, and Mindanao; in Mindanao recorded only in Northern Mindanao region (Cabras et al. 2016a).

Literature data for Northern Mindanao. Misamis Oriental province: Gingoog City (Cabras et al. 2016a).

Habitat. Forest, arboreal species found on vertical and fallen tree trunks.

Tricondyla (Stenotricondyla) cavifrons Schaum, 1862

Figure 6E

General distribution. Species endemic to the Philippines where it was noted from Balabac, Mindanao, and Palawan; in Mindanao it was recorded only from Bangsamoro Autonomous Region in Muslim Mindanao (Marohomsalic et al. 2021), this is the first record from Northern Mindanao region.

Material examined. Bukidnon province: Mt. Kitanglad, 10.2014, 1, leg. N. Mohagan (JWC); Mt. Kitanglad, 11-12.2014, $2 \hat{\jmath} \hat{\jmath} 1$, , leg. N. Mohagan (JWC);

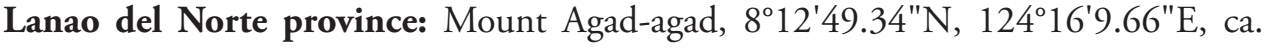
470 m a.s.l., 11.2019, 3 exx., leg. J. Ebina, M. L. Lumontod, G. C. Café (RJC).

Habitat. Forest, arboreal species found on vertical and fallen tree trunks. Marohomsalic et al. (2021) recorded native and invasive tree species having extrafloral nectaries as favorite hunting areas for this species.

\section{Genus Neocollyris Horn, 1901}

The genus Neocollyris is represented in the Philippines by 29 species, including 12 recorded from Mindanao Island (Naviaux 1994; Cabras et al. 2016a).

\section{Subgenus Neocollyris Horn, 1901}

Neocollyris (Neocollyris) albitarsis (Erichson, 1834)

Figure 6F

General distribution. Species endemic to the Philippines, noted from Homonhon, Luzon, Mindanao, and Palawan; in Mindanao it was recorded only from Northern 
Mindanao and Bangsamoro Autonomous Region in Muslim Mindanao region (Cabras et al. 2016a; Marohomsalic et al. 2021).

Literature data for Northern Mindanao. Bukidnon province: Impasung-ong (Cabras et al. 2016a).

Habitat. Forest, arboreal species found on vertical and fallen tree trunks and leaves of different bush species.

Neocollyris (Neocollyris) brevicula Naviaux, 1994

Figure $7 \mathrm{~A}$

General distribution. Species endemic to Philippines, known from Basilan, Mindanao, and Samar island (Cabras et al. 2016a); in Mindanao recorded only in Northern Mindanao (Naviaux 1994).

Literature data for Northern Mindanao. Lanao del Norte province: Municipality of Balo-i, Barangay Momungan (Naviaux 1994).

Habitat. Forest, arboreal species found on vertical and fallen tree trunks and leaves of different bush species.

Neocollyris (Neocollyris) emarginata (Dejean, 1825)

Figure 7B

General distribution. Species noted from Borneo, Indonesia, Malaysia, and Philippines, where it was found in Mindanao and Palawan; in Mindanao recorded only in Northern Mindanao region (Cabras et al. 2016a).

Literature data for Northern Mindanao. Bukidnon province: Impasug-ong (Cabras et al. 2016a).

Habitat. Forest, arboreal species found on vertical and fallen tree trunks and leaves of different bush species.

Subgenus Heterocollyris Naviaux, 1995

Neocollyris (Heterocollyris) affinis (Horn, 1892)

Figure 7C

General distribution. Species endemic to the Philippines where it was recorded in Bohol, Leyte, Luzon, Mindanao, Panay, and Samar; in Mindanao found only in Northern Mindanao region (Cabras et al. 2016a).

Literature data for Northern Mindanao. Bukidnon province: Impasug-ong (Cabras et al. 2016a); Misamis Oriental province: Balatucan-Lumot (Cabras et al. 2016a).

Habitat. Forest, arboreal species found on vertical and fallen tree trunks and leaves of different bush species. 


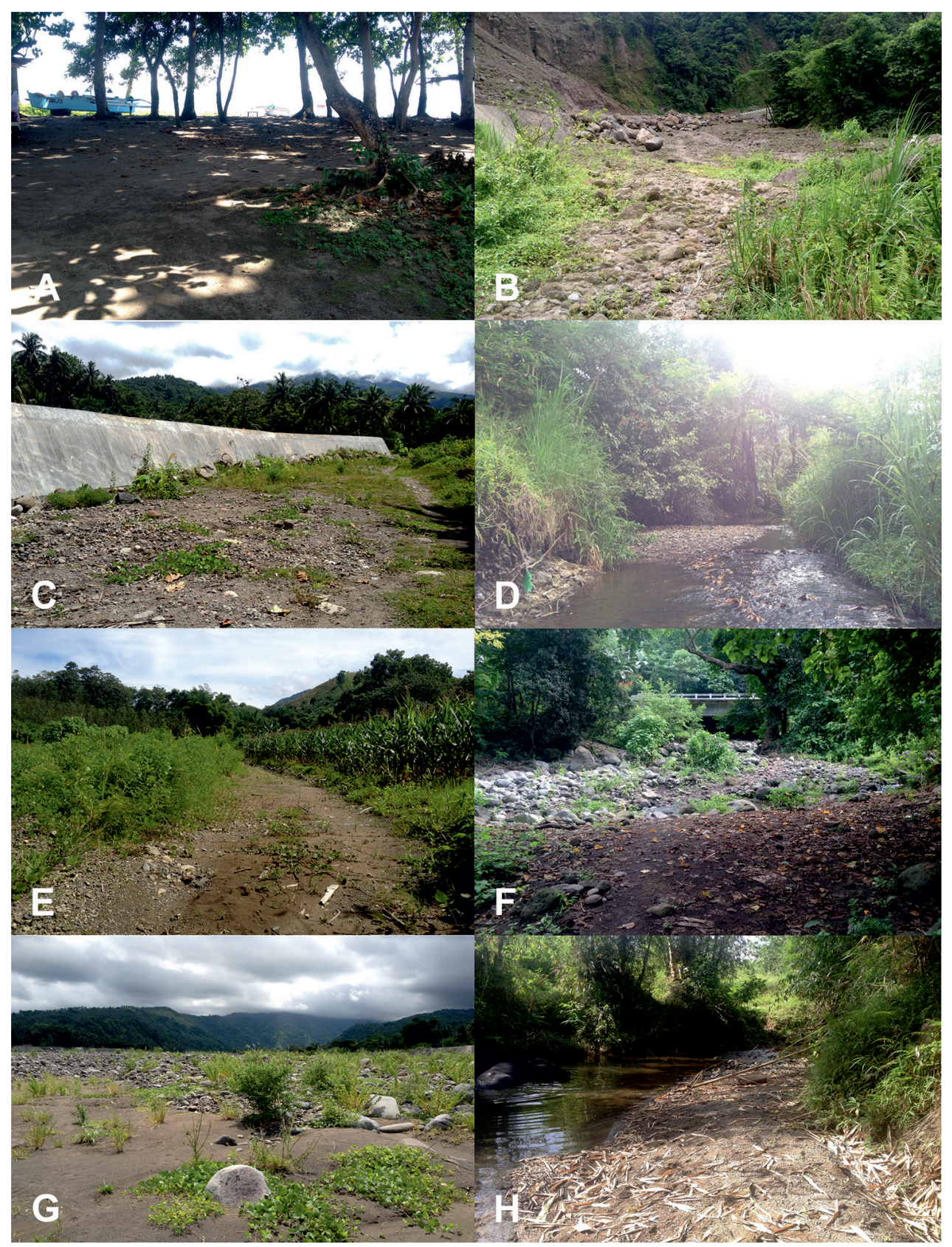

Figure 2. Habitats of tiger beetles from Northern Mindanao region: Calomera mindanaoensis (A-G) (sites 1-7), Cylindera discreta elaphroides $(\mathbf{H})$ (site 8), C. minuta $(\mathbf{G})$ (site 7), C. mouthiezi $(\mathbf{H})$ (site 8) (descriptions of sites in Table 1; photographs DAPA). 


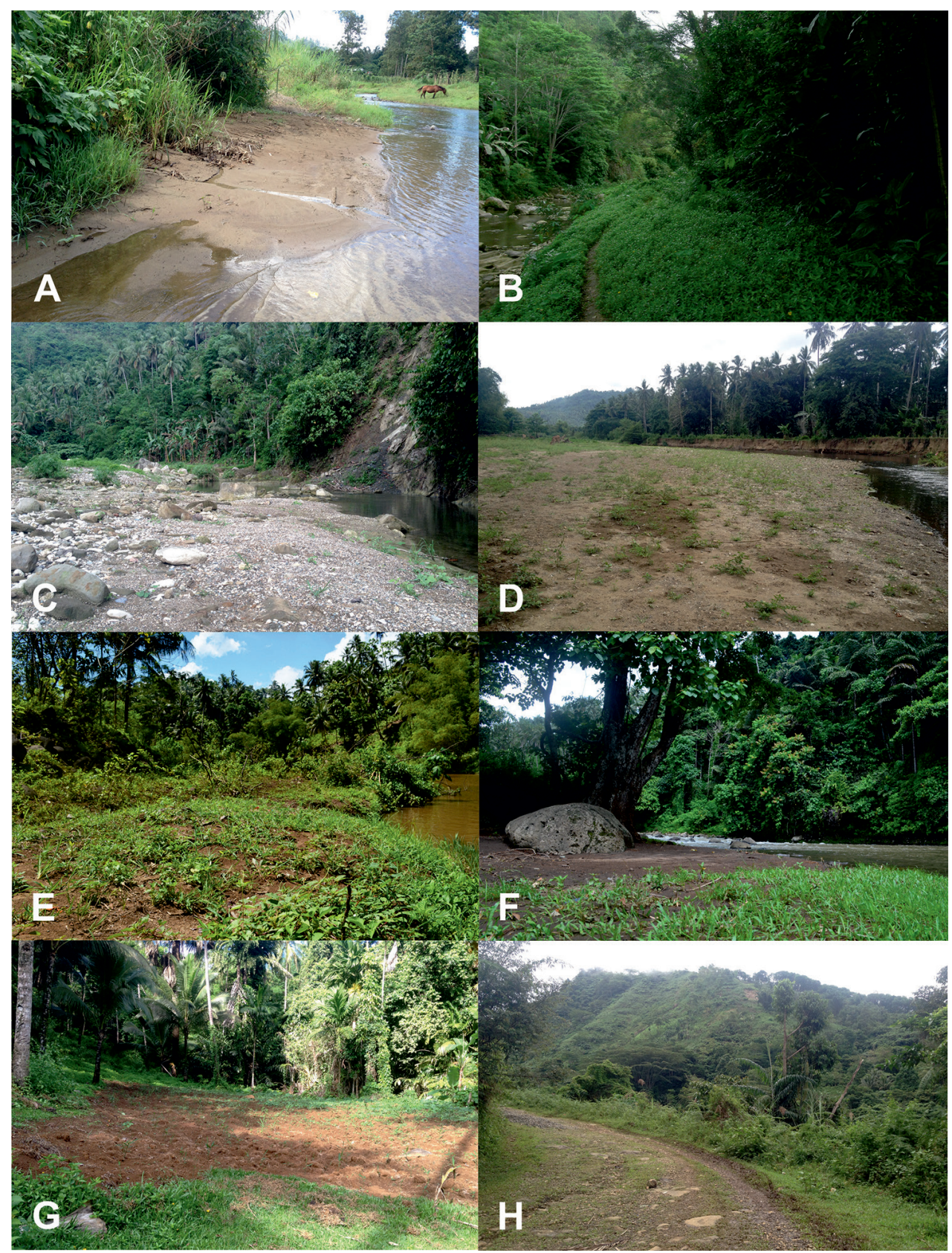

Figure 3. Habitats of tiger beetles from Northern Mindanao region: Calomera angulata angulata (C-E) (sites 10-12), C. lacrymosa (C, E) (sites 10, 12), C. mindanaoensis (C-F, H) (sites 10-13, 15), Cylindera discreta elaphroides (A, C-E) (sites 9-12), C. minuta (A, C-E) (sites 9-12), C. viduata (G) (site 14), Lophyra striolata tenuiscripta (G) (site 14), Therates coracinus coracinus (B) (site 10) (descriptions of sites in Table 1; photographs A, C-H DAPA, B RJ). 
Neocollyris (Heterocollyris) similior (Horn, 1893)

Figures 4D, 7D

General distribution. Species endemic to Philippines where it was recorded only from Mindanao (Naviaux 1994; Cabras et al. 2016; Marohomsalic et al. 2021).

Material examined. Misamis Oriental province: Mimbilisan Protected Landscape, $8.94884 \mathrm{~N}, 124.86517 \mathrm{E}, 501 \mathrm{~m}$ a.s.l., 18.07.2017, 10, leg. O. Bagona (RJC); Lanao del Norte province: Dodiongan Falls, Iligan City - Barangay Bonbonon, 8.271457N, 124.314140E, 47 m a.s.l., 11.2019., 1 ex., leg. M. L. Lumontod (RJC).

Habitat. Forest, arboreal species found on vertical and fallen tree trunks and leaves of different bush species.

Neocollyris (Heterocollyris) speciosa (Schaum, 1863)

Figure $7 \mathrm{E}$

General distribution. Species endemic to the Philippines, where it was noted only from Luzon, Mindoro (Cabras et al. 2016a), and Mindanao (new record).

Material examined. Bukidnon province: Mt. Kitanglad, 11-12.2014, 2 $2+q$, leg. N. Mohagan (JWC).

Habitat. Forest, arboreal species found on vertical and fallen tree trunks and leaves of different bush species.

Genus Protocollyris Mandl, 1975

Protocollyris mindanaoensis (Mandl, 1974)

Figure $7 \mathrm{~F}$

General distribution. Species endemic for Philippines where it was noted only from Mindanao Island (Cabras et al. 2016a) from Northern Mindanao region (Naviaux 1994).

Literature data for Northern Mindanao. Lanao del Norte province: Momungan [actually Barangay Momungan in Municipality of Balo-i] (Naviaux 1994).

Habitat. Forest, arboreal species.

Tribe Cicindelini Latreille, 1802

Subtribus Theratina Horn, 1910

Genus Therates Latreille, 1817

The genus Therates is represented in the Philippine islands by six species, including four or five recorded from Mindanao Island; three of them have been noted from the Northern Mindanao region by Cabras et al. (2016a). 


\section{Therates coracinus coracinus Erichson, 1834}

Figures 3B, 4D, 8A

General distribution. Subspecies known from Indonesia, Moluccas, and Philippines, where it was recorded in Balabac, Leyte, Luzon, Mindanao, Mindoro, Negros, Palawan, Panay, Romblon, and Samar; in Mindanao recorded from Davao, Northern Mindanao, and Soccsksargen regions (Wiesner 1988; Cabras et al. 2016a, b; Cabras and Wiesner 2016; Pepito et al. 2020).

Literature data for Northern Mindanao. Bukidnon province: Impasug-ong (Cabras et al. 2016a).

Material examined. Bukidnon province: Mt. Kitanglad, 28.07.1990, $1 \overbrace{}^{\Uparrow} 1 q$, ex

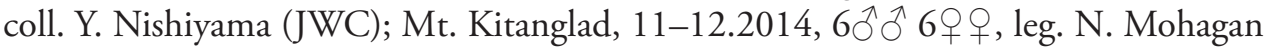
(JWC); Mt. Kalatungan, Sitio Bato, Municipality of Maramag, 11.2019, 1 ex., leg. R. Jaskuła (RJC). Lanao del Norte province: Dodiongan Falls, Iligan City - Barangay Bonbonon, 8.271457N, 124.314140E, $47 \mathrm{~m}$ a.s.l., on Araceae leaves, 07.12.2018, 10̂, leg. R. Jaskuła (RJC), 11.2019, 6 exx., leg. D. A. P. Acal, J. Ebina, M. L. Lumontod, R. Jaskuła (RJC); Mount Agad-agad, 8 $12^{\prime} 49.34 " \mathrm{~N}, 124^{\circ} 16^{\prime} 9.66^{\prime \prime E}$, ca. $470 \mathrm{~m}$ a.s.l., on leaves, 11.2019, 2 exx., D. A. P. Acal, J. Ebina (RJC). Misamis Oriental province: Mimbilisan Protected Landscape, 8.94884N, 124.86517E, 501 m a.s.l., 18.07.2017, 3 exx., leg. O. Bagona (RJC); Bolyok Falls, Barangay Lubilan, Naawan Municipality, 11.20218, 4 exx., leg. R. Jaskuła, D.A.P. Acal, J. Ebina, M. L. Lumontod (RJC); Mambuntan Falls, Barangay Lubilan, Naawan Municipality, 8.412300N, 124.351642E, 11.2019, 1 ex., leg. R. Jaskuła (RJC).

Habitat. Forest species noted on tree trunks and leaves.

Remarks. When disturbing, actively fast flying among trees; during flight shows bright orange abdomen coloration. Taxonomical status of both subspecies of Therates coracinus noted in Mindanao (spp. coracinus and ssp. fulvescens Wiesner, 1988) should be revised including molecular data as they probably represent separate species or synonyms.

\section{Therates fasciatus (Fabricius, 1801)}

\section{Therates fasciatus fasciatus (Fabricius, 1801)}

Figures 4D, 8B

General distribution. Subspecies known from Indonesia and Philippines. In the Philippines recorded only from Mindanao and Palay islands (Cabras et al. 2016a); from Mindanao Island it was known only from Davao and Northern Mindanao regions (Cabras et al. 2016b).

Literature data for Northern Mindanao. Bukidnon province: [no detailed locality] (Wiesner 1988).

Material examined. Bukidnon province: [no detailed locality], 1977, 1 9 , leg. R. Lumawig (JWC). Camiguin province: Camiguin Island, Municipality of Catarman, Mt. Timpoong-Hibok-Hibok Natural Monument, Mt. Hibok-Hibok 11.2019, 2 exx., 


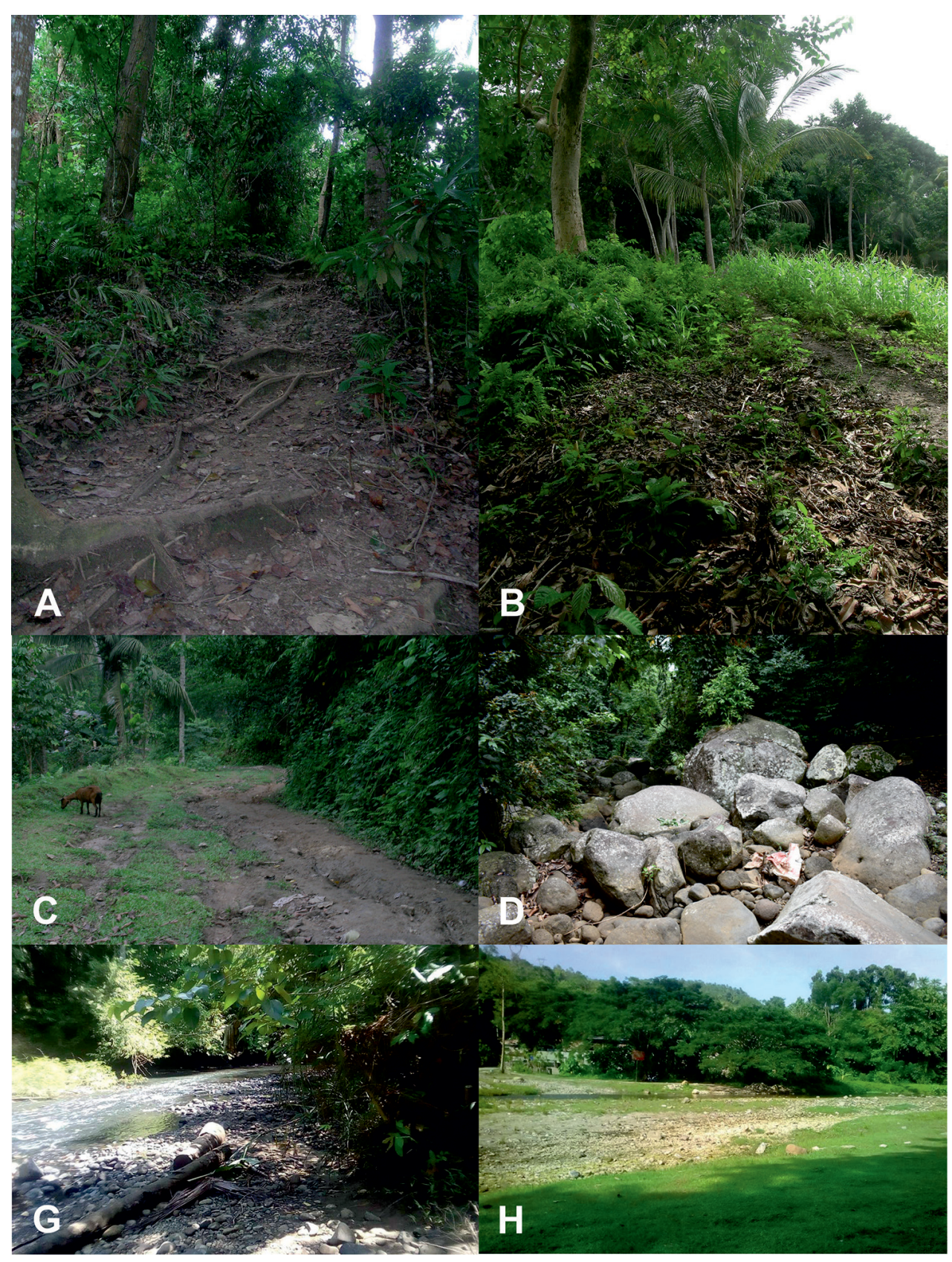

Figure 4. Habitats of tiger beetles from Northern Mindanao region: Calomera mindanaoensis (C) (site 16) (E, F) (sites 18-19), Prothyma heteromallicollis heteromallicollis (A) (site 16), Neocollyris similior (D) (site 17), Therates coracinus coracinus (D) (site 17), T. fasciatus fasciatus (D) (site 17), and Tricondyla aptera punctipennis (B) (site 16) (descriptions of sites in Table 1; photographs A-C RJ, D O. Bagona, E A.B. Lapore, F C. Torres). 


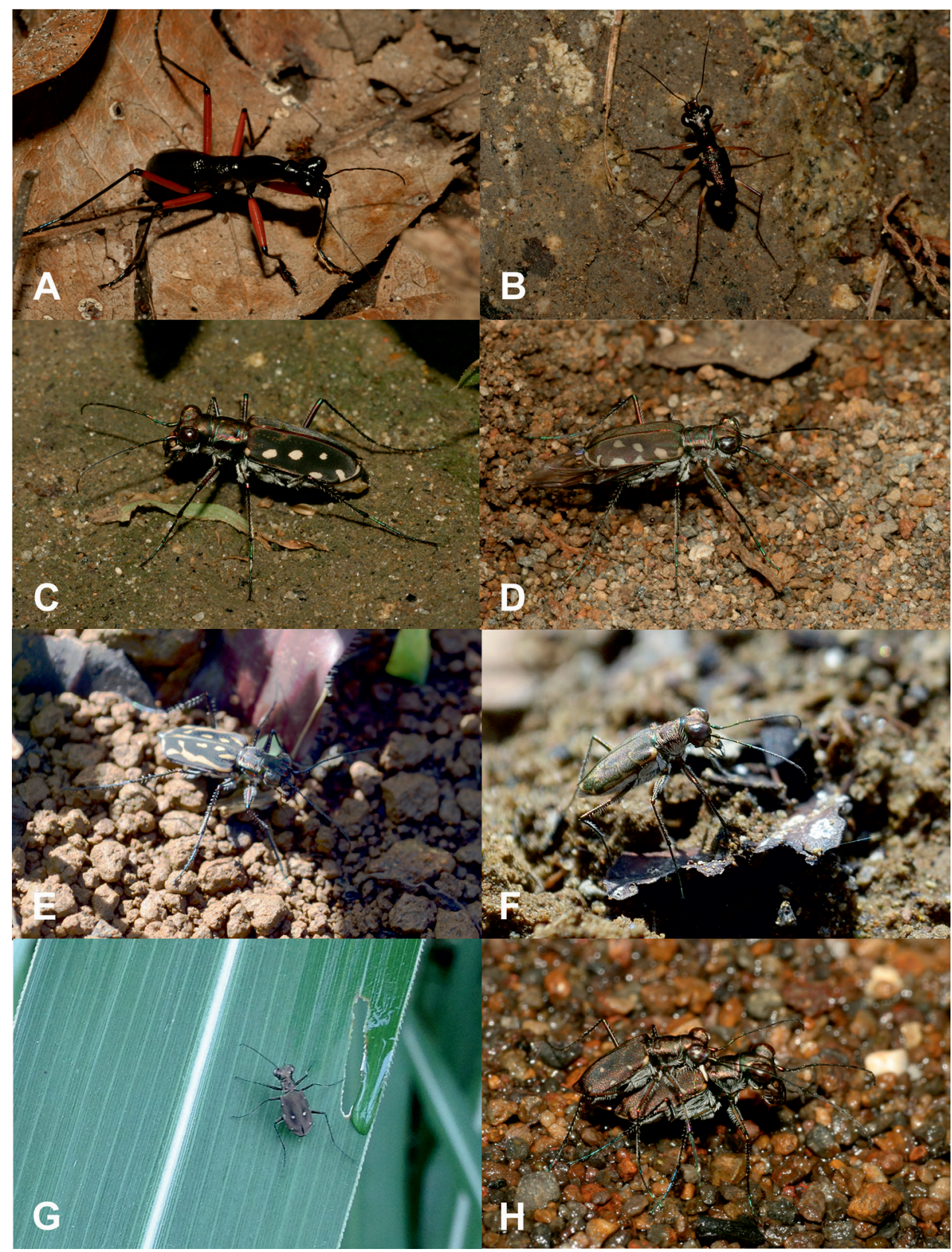

Figure 5. Some of the tiger beetle species recorded in Northern Mindanao region: A Tricondyla (Tricondyla) aptera punctipennis B Prothyma (Symplecthyma) heteromallicollis heteromallicollis $\mathbf{C}$ Calomera mindanaoensis D C. lacrymosa $\mathbf{E}$ Lophyra (Spilodia) striolata tenuiscripta $\mathbf{F}$ Cylindera (Eugrapha) minuta $\mathbf{G}, \mathbf{H}$ C. (Ifasina) discreta elaphroides (photographs A-D, H RJ, E-G DAPA). 
leg. R. Jaskuła, D. A. P. Acal (RJC); Lanao del Norte province: Dodiongan Falls, Iligan City - Barangay Bonbonon, 8.271457N, 124.314140E, 47 m a.s.l., 11.2019, 11 exx., leg. R. Jaskuła, D.A.P. Acal, J. Ebina, M. L. Lumontod (RJC); near Sikyop Cave, Iligan City - Barangay Lawlawon, 8.246627N, 124.422387E, 11.2019, 2 exx., leg. R. Jaskuła (RJC); Misamis Oriental province: Mimbilisan Protected Landscape $8.94884 \mathrm{~N}$, 124.86517E, 501 m a.s.l., 18.07.2017, 13 exx., leg. O. Bagona (RJC); Bolyok Falls, Barangay Lubilan, Naawan Municipality, 11.2019, 1 ex., leg. R. Jaskuła (RJC).

Habitat. Forest species noted on tree trunks and leaves.

Therates fasciatus pseudolatreillei Horn, 1928

Figure 8B

General distribution. Subspecies endemic to Philippines where it was recorded from Leyte, Mindanao, and Mindoro; in Mindanao noted only from Northern Mindanao and Soccsksargen regions (Wiesner 1988; Cabras et al. 2016a; Pepito et al. 2020).

Literature data for Northern Mindanao. Bukidnon province: Impasug-ong (Cabras et al. 2016a); Lanao del Norte province: "Mai-nit" (actually Barangay Mainit) (Wiesner 1988); Misamis Occidental province: Malindang Range (Cabras et al. 2016a).

Material examined. Bukidnon province: Mt. Talemo, 30.06.1977, 1ð઼, leg. M. Sato (JWC); Lanao del Norte province: Barangay Mainit (between Iligan City and Cagayan de Oro), 17-20.08.1978, 2ð⿱ 2 2우, leg. Cabides et Lobin (JWC); Misamis

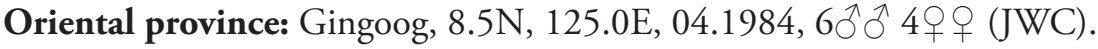

Habitat. Forest species noted on tree trunks and leaves.

Remarks. Taxonomical status of all four subspecies of Therates fasciatus noted in Mindanao (spp. fasciatus (Fabricius, 1801), spp. quadrimaculatus Horn, 1895, spp. pseudolatreillei Horn, 1928, and ssp. flavohumeralis Mandl, 1964) should be revised including molecular data as probably at least some represent separate species or synonyms.

\section{Therates fulvipennis Chaudoir, 1848}

\section{Therates fulvipennis bidentatus Chaudoir, 1861}

Figure 8C

General distribution. Subspecies known from Indonesia and Philippines (Basilan and Mindanao islands); from Mindanao noted from Northern Mindanao region only (Wiesner 1988; Cabras et al. 2016a).

Literature data for Northern Mindanao. Lanao del Norte province: "Ma-Init" [actually Barangay Mainit] (Wiesner 1988).

Material examined. Bukidnon province: Mt. Kitanglad, 10.2014, $15 \hat{\jmath}$ 5 우우, leg. N. Mohagan (JWC); Lanao del Norte province: Barangay Mainit (between Iligan City and Cagayan de Oro), 17-20.08.1978, $50 \hat{\jmath} 3$ 우우, leg. Cabides et Lobin (JWC).

Habitat. Forest species noted on tree trunks and leaves. 


\section{Therates fulvipennis everetti Erichson, 1834}

Figure 8C

General distribution. Subspecies endemic to the Philippines, where it was recorded from Dinagat, Luzon, Mindanao, Negros, and Panay; in Mindanao it was recorded in Davao, Northern Mindanao, and Soccsksargen regions (Cabras et al. 2016a, b).

Literature data for Northern Mindanao. Bukidnon province: Impasug-ong (Cabras et al. 2016a).

Habitat. Forest species noted on tree trunks and leaves.

Remarks. Taxonomical status of all three subspecies of Therates fulvipennis noted in Mindanao (spp. bidentatus Chaudoir, 1861, ssp. fulvipennis Chaudoir, 1848, and spp. everetti Erichson, 1834) should be revised including molecular data as probably at least some of them represent separate species or synonyms.

\section{Subtribus Dromicina Thomson, 1859}

\section{Genus Prothyma Hope, 1838}

The genus Prothyma is represented in the Philippine islands by 12 species, including six recorded from Mindanao Island (Cabras et al. 2016a).

\section{Prothyma (Symplecthyma) heteromallicollis heteromallicollis Horn, 1909}

Figures 4A, 5B, 8D

General distribution. Species endemic to the Philippines, where it was recorded on Luzon and Mindanao till now (Cabras et al. 2016a); in Mindanao it has been noted from Davao (Cabras et al. 2016b) and Northern Mindanao (this publication) regions.

Material examined. Bukidnon province: Mt. Kitanglad, 11-12.2014, $3 \hat{\delta} \widehat{\partial}$ 1 , , leg. N. Mohagan (JWC); Lanao del Norte province: Mount Agad-agad, $8^{\circ} 12^{\prime} 49.34^{\prime \prime N}, 124^{\circ} 16^{\prime} 9.66^{\prime \prime E}, 470 \mathrm{~m}$ a.s.l., 18.10.2018, 10 exx., leg. D. A. P. Acal

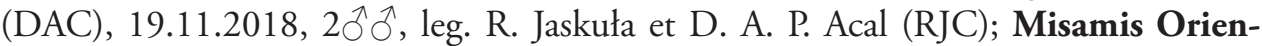
tal province: Mimbilisan Protected Area, 8.94884N, 124.86517E, $501 \mathrm{~m}$ a.s.l., 18.07.2017, 10, leg. O. Bagona (RJC).

Habitat. Species found on shaded forest paths and forest litters.

Remarks. Some individuals were observed resting on undershrub leaves along forest trails.

\section{Genus Heptodonta Hope, 1838}

The genus Heptodonta is represented in the Philippine islands by five species, including two recorded from Mindanao Island (Cabras et al. 2016a; Görn 2020). 

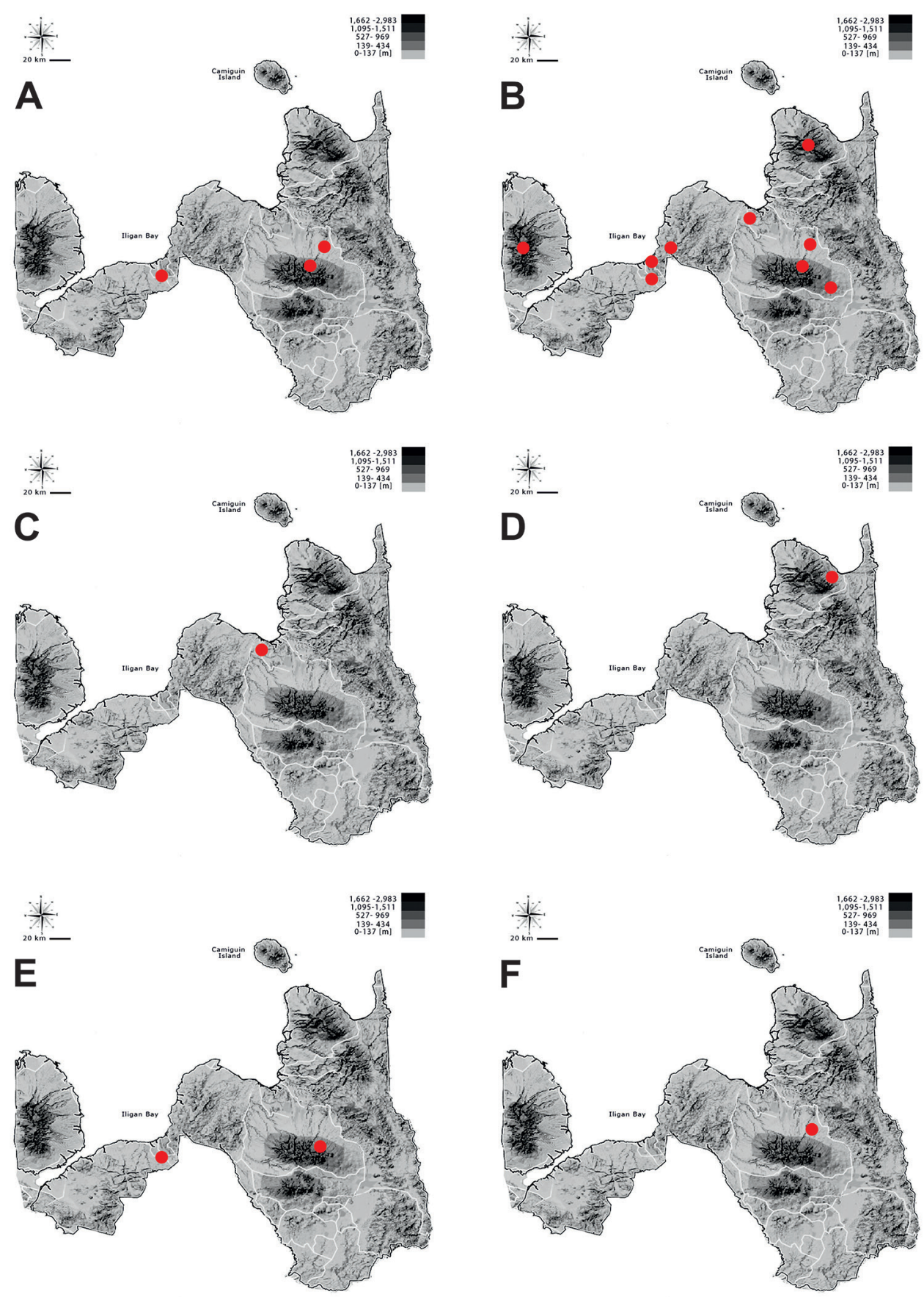

Figure 6. Distribution of A Tricondyla (Tricondyla) aptera punctipennis B T. (T.) elongata C T. (T.) gracilis D T. (Stenotricondyla) cyanipes E T. (Stenotricondyla) cavifrons, and $\mathbf{F}$ Neocollyris (Neocollyris) albitarsis in Northern Mindanao region. 

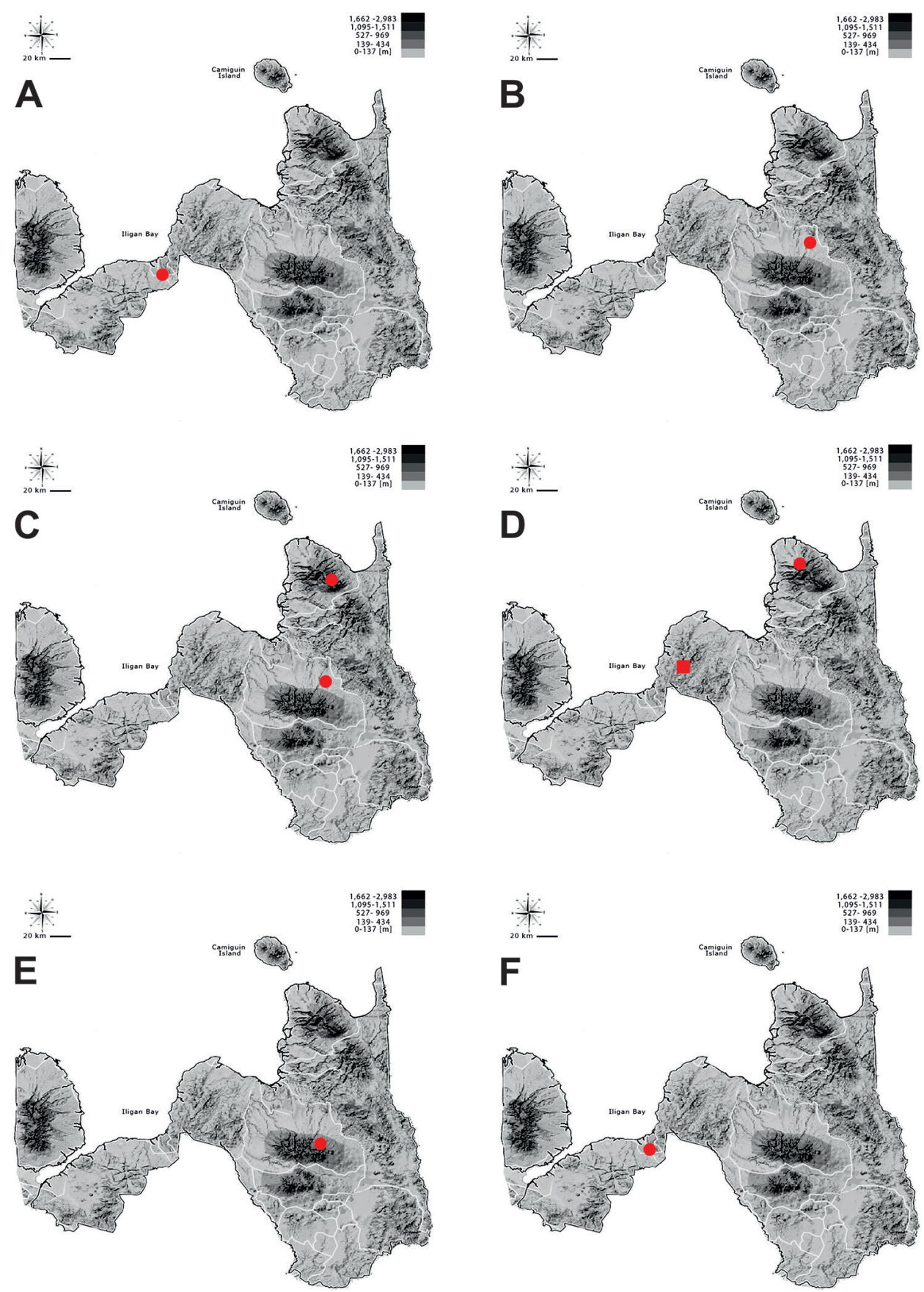

Figure 7. Distribution of A Neocollyris (Neocollyris) brevicula B $N$. (N.) emarginata C Neocollyris (Heterocollyris) affinis $\mathbf{D} N$. (Heterocollyris) similior $\mathbf{E} N$. (Heterocollyris) speciosa, and $\mathbf{F}$ Protocollyris mindanaoensis in Northern Mindanao region. 
Heptodonta nigrosericea (W. Horn, 1930)

Figure 8E

General distribution. Species endemic to the Philippines, where it has been noted on Mindanao and Panay islands only; in Mindanao it was noted in Davao, North Mindanao, and Soccsksargen regions (Cabras et al. 2016a; Cabras and Wiesner 2016; Görn 2020; Medina et al. 2020c; Medina 2020; Pepito et al. 2020).

Literature data for Northern Mindanao. Bukidnon province: Impasug-ong (Cabras et al. 2016a: noted as Heptodonta lumawigi Wiesner, 1980); Mt. Kintanglad (Görn 2020); Kabanglasan [= Cabanglasan] (Görn 2020); Intavas (Görn 2020); Silipon (Görn 2020); Misamis Occidental province: Mt. Malindang Range (Cabras et al. 2016a: noted as H. lumawigi Wiesner, 1980); Misamis Oriental province: Mt. Balatucan-Lumot (Cabras et al. 2016a: noted as H. lumawigi Wiesner, 1980); Gingoog (Görn 2020).

Material examined. Bukidnon province: Mt. Kintanglad, 10.2014, $5 \hat{\jmath} \hat{\delta} 5$ 우, leg. N. Mohagan (JWC); [no detailed locality], 1977, 1q, leg. R. Lumawig (JWC); Misamis Oriental province: Gingoog, 8.5N, 125.0E, 04.1984, 7 $\widehat{\jmath} \hat{\jmath} 13$ 우 (JWC).

Habitat. Species noted in shaded areas on river banks.

Remarks. Heptodonta lumawigi is a junior synonym of this species (Görn 2020).

\section{Subtribe Cicindelina Latreille, 1802}

\section{Genus Calomera Motschulsky, 1862}

The genus Calomera is represented in the Philippine islands by five species, including four recorded from Mindanao Island (Cabras et al. 2016a). Three of them have been noted from the Northern Mindanao region.

\section{Calomera angulata angulata (Fabricius, 1798)}

Figures 3C-E, 8F

General distribution. India, Nepal, Sri Lanka, Thailand, Pakistan, Afghanistan, Cambodia, Vietnam, Laos, Taiwan, Malaysia, Indonesia, China; in the Philippines the species was recorded only from Luzon (Cabras et al. 2016a) and Mindanao (new record).

Material examined. Lanao del Norte province: Iligan City - Barangay Merila,

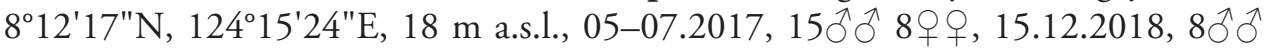
3 우, leg. D. A. P. Acal (DAC); Iligan City - Barangay Bonbonon, 8.265458N, 124.310138E, $47 \mathrm{~m}$ a.s.l., 05-07.2017, 3ठำ 1q, leg. D. A. P. Acal (DAC); Municipality of Bacolod, Barangay Esperanza, $8^{\circ} 10^{\prime} 12^{\prime \prime} \mathrm{N}, 124^{\circ} 0^{\prime} 22^{\prime \prime} \mathrm{E}, 27 \mathrm{~m}$ a.s.l., $05-$ 07.2017, 1 q , leg. D. A. P. Acal (DAC); Iligan City - Barangay Puga-an, sandy bank of Puga-an River, $8^{\circ} 13^{\prime} 21.3^{\prime \prime N}, 124^{\circ} 15^{\prime} 52.0^{\prime \prime E}, 29.10 .2018,3{ }^{\lambda}{ }^{\top} 6$ 6우, leg. C. Torres (RJC); Iligan City - Barangay Puga-an, sandy bank of Puga-an River, 8¹3'29.6"N, 


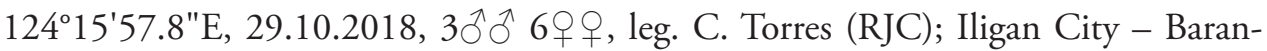
gay Puga-an, rocky bank of Puga-an River, $8^{\circ} 13^{\prime} 31.5^{\prime \prime N}, 124^{\circ} 16^{\prime} 08.6 " \mathrm{E}, 29.10 .2018$,

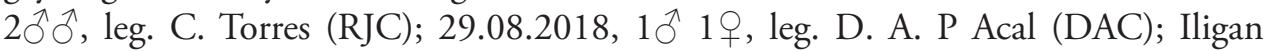

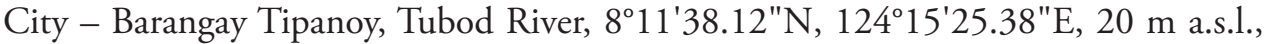

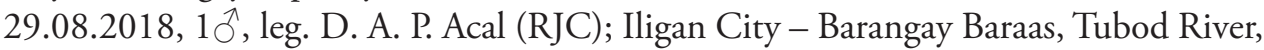
$8^{\circ} 12^{\prime} 40.23^{\prime \prime} \mathrm{N}, 124^{\circ} 14^{\prime} 53.25^{\prime \prime E}, 12 \mathrm{~m}$ a.s.l., 17.07.2018, $40^{\top} \mathrm{o}^{\top} 1$ \% leg. D. A. P. Acal (RJC); Barangay Merila, Iligan City, 15.12.2018, $8 \hat{\jmath} 3$ 3 + , leg. D. A. P. Acal (RJC).

Habitat. The species occurs on sandy river banks exposed to direct sunlight (pers. obs.).

Remarks. First records both from Northern Mindanao region and entire Mindanao Island. This species was observed to co-occur with Calomera mindanaoensis, $C$. lacrymosa, Cylindera discreta elaphroides, and C. minuta.

\section{Calomera cabigasi Cassola, 2011}

Figure 9A

General distribution. Species endemic to Philippines where it was found only in Mindanao (Northern Mindanao region) (Cassola 2011; Cabras et al. 2016a).

Literature data for Northern Mindanao. Bukidnon province: Impasug-ong (Cassola 2011); Misamis Oriental province: Gingoog City (Cassola 2011).

Habitat. Species noted from river banks.

\section{Calomera lacrymosa (Dejean, 1825)}

Figures 3C, E, 5D, 9B

General distribution. Species endemic to Philippines where it was found in greater part of the country, including Balabac, Bucas Grande, Homonhon, Luzon, Palawan, Mindanao, Mindoro, and Sibuyan; in Mindanao Island it was noted from Davao and Northern Mindanao regions (Cabras et al. 2016a, b).

Literature data for Northern Mindanao. Lanao del Norte province: Iligan City, Barangay Tipanoy (Cassola 2000; Cabras et al. 2016a); Misamis Oriental province: Municipality of Tagoloan, Tagoloan River (Cassola 2000; Cabras et al. 2016a).

Material examined. Lanao del Norte province: Iligan City - Barangay Bonbonon, 8.265458N, 124.310138E, $47 \mathrm{~m}$ a.s.l., 05-07.2017, 40 exx., leg. D. A. P. Acal (DAC); Municipality of Bacolod, Barangay Esperanza, $8^{\circ} 10^{\prime} 12^{\prime \prime} \mathrm{N}, 124^{\circ} 0^{\prime} 22^{\prime \prime} \mathrm{E}$, 27 m a.s.l., 05-07.2017, 210 exx., leg. D. A. P. Acal (DAC), 13.12.2018, 4ภํ, leg. R. Jaskuła (RJC); Iligan City - Barangay Puga-an, sandy bank of Puga-an River,

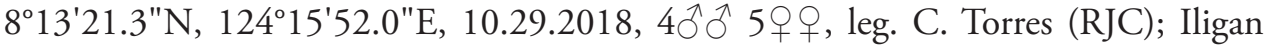
City - Barangay Puga-an, sandy bank of Puga-an River, $8^{\circ} 13^{\prime} 29.6^{\prime \prime N}, 124^{\circ} 15^{\prime} 57.8^{\prime \prime} \mathrm{E}$,

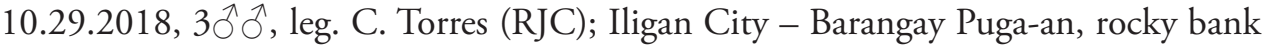
of Puga-an River, $8^{\circ} 13^{\prime} 31.5^{\prime \prime} \mathrm{N}, 124^{\circ} 16^{\prime} 08.6^{\prime \prime E}, 10.29 .2018,7 \delta^{\wedge} 1$ 잉 leg. C. Tor-

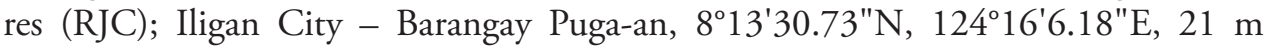



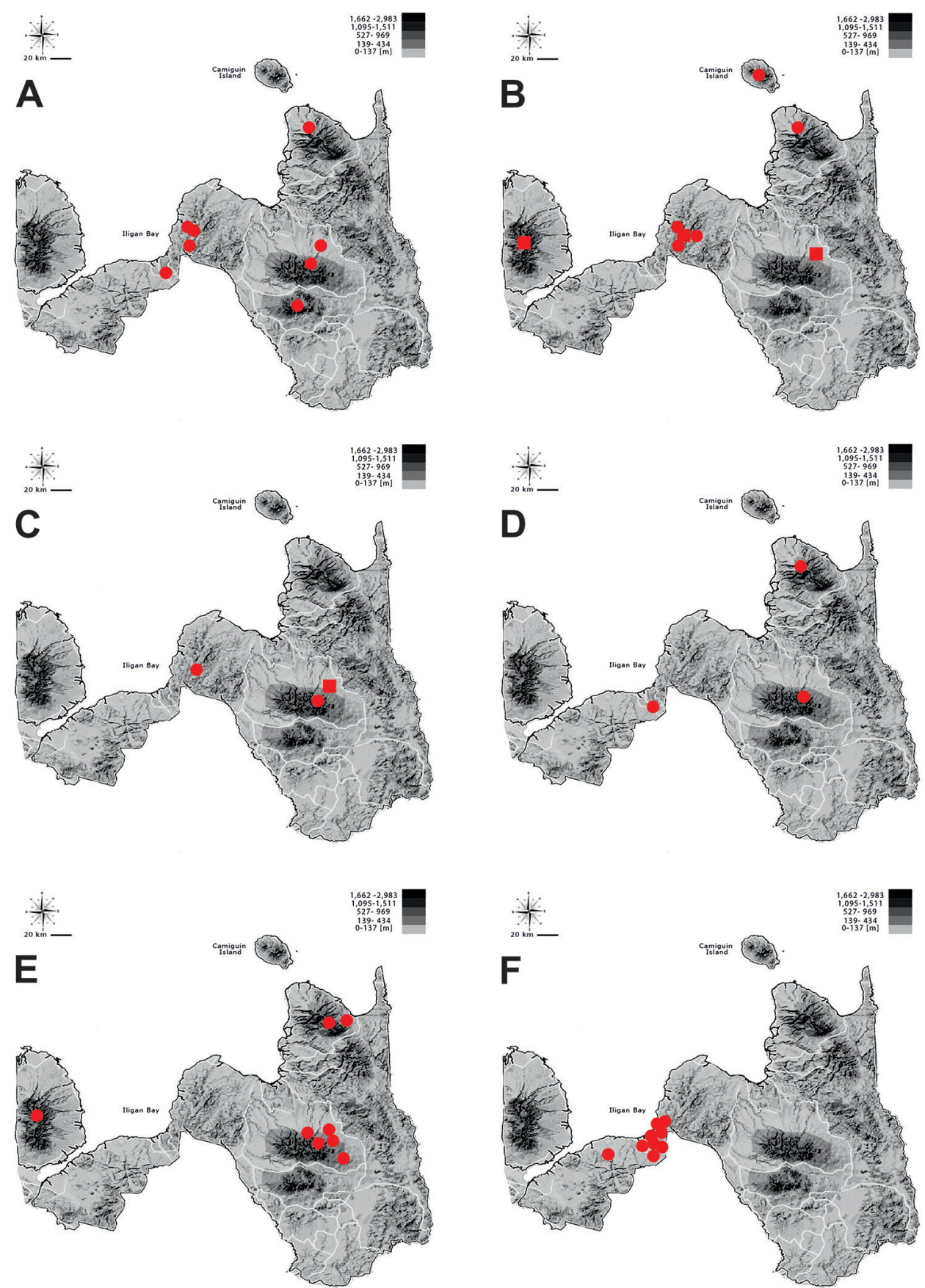

Figure 8. Distribution of A Therates coracinus coracinus B Therates fasciatus fasciatus (circle) and T. fasciatus pseudolatreillei (square) C Therates fulvipennis bidentatus (cirle) and T. fulvipennis everetti (square) D Prothyma (Symplecthyma) heteromallicollis heteromallicollis $\mathbf{E}$ Heptodonta nigrosericea, and $\mathbf{F}$ Calomera angulata angulata in Northern Mindanao region. 

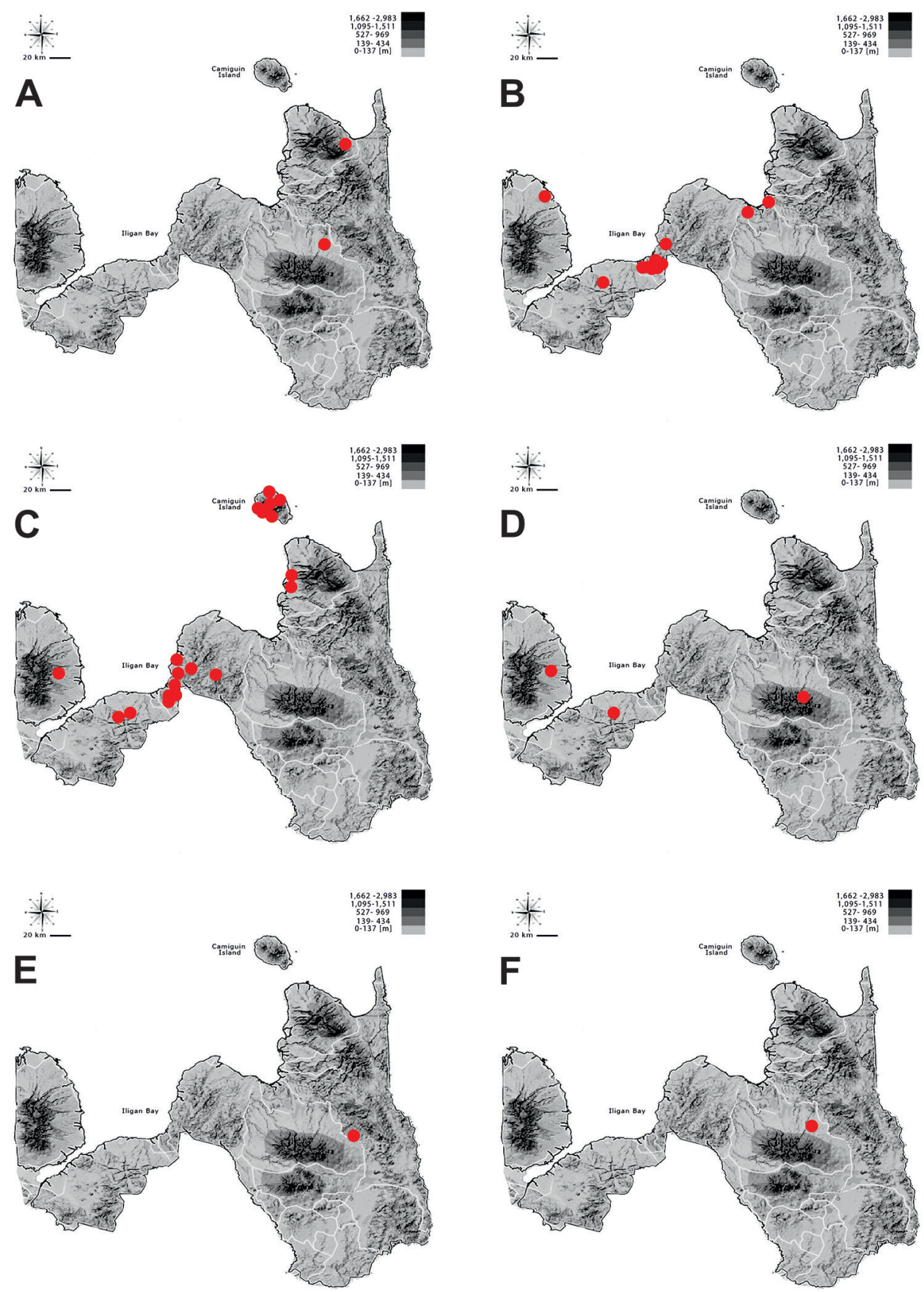

Figure 9. Distribution of A Calomera cabigasi B C. lacrymosa C C. mindanaoensis D Lophyra (Spilodia) striolata tenuiscripta $\mathbf{E}$ Thopeutica (Thopeutica) angulihumerosa, and $\mathbf{F}$ T. (T.) darlingtonia in Northern Mindanao region. 
a.s.l., $3 \widehat{\jmath}$, leg. D. A. P. Acal (RJC); Iligan City - Barangay Tipanoy, Tubod River, $8^{\circ} 11^{\prime} 38.12^{\prime \prime N}, 124^{\circ} 15^{\prime} 25.38^{\prime \prime E}, 20$ m a.s.l., 29.08.2018, 1 \% , leg. D. A. P. Acal (RJC); Iligan City - Barangay Merila, $8^{\circ} 12^{\prime} 17^{\prime \prime} \mathrm{N}, 124^{\circ} 15^{\prime} 24^{\prime \prime} \mathrm{E}, 13.12 .2018,18 \mathrm{~m}$ a.s.l.,

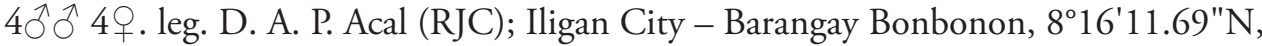
$124^{\circ} 17^{\prime} 16.11$ "E, $11 \mathrm{~m}$ a.s.l., 04.11.2018, 40 exx., leg. D. A. P. Acal (DAC); Baran-

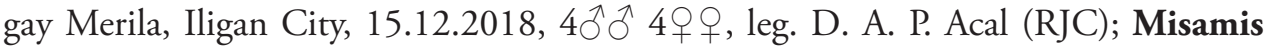

Oriental province: Cagayan de Oro City, Malasag Cugman, Mapawa Nature Park,

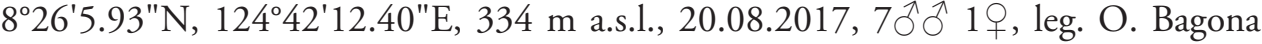
(RJC); Misamis Occidental province: Municipality of Lopez Jaena, $8^{\circ} 33^{\prime} 00^{\prime N}$,

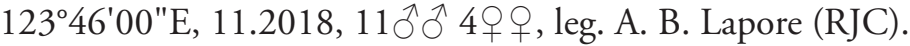

Habitat. The species occurs on sandy river banks (pers. obs.).

Remarks. At least in some areas Calomera lacrymosa seems to occur sympatrically or even syntopically with $C$. mindanaoensis (pers. obs.). C. lacrymosa was recently noted as a host of Hexathrombium parasitic mites (Acari: Microtrombidiidae) (Acal et al. in press).

Calomera mindanaoensis (Cassola, 2000)

Figures $2 \mathrm{~A}-\mathrm{G}, 3 \mathrm{C}-\mathrm{F}, \mathrm{H}, 4 \mathrm{C}, 5 \mathrm{C}, 9 \mathrm{C}$

General distribution. Species endemic to Philippines where it was found in Mindanao (Cassola 2000; Cabras et al. 2016a, b; Cabras and Wiesner 2016) and Camiguin islands (new record); till now in Mindanao it was recorded in Davao, Northern Mindanao, Soccsksargen, and Zamboanga Peninsula regions (Cassola 2000; Cabras et al. 2016a, b; Cabras and Wiesner 2016; Pepito et al. 2020).

Literature data for Northern Mindanao. Bukidnon province: S. Vicente, 20 km S Cagayan de Oro (Cassola 2000); Impasug-ong (Cabras et al. 2016a); Lanao del Norte province: Iligan City, Tipanoy (Cassola 2000); Misamis Oriental province: Tagoloan River, Tagoloan (Cassola 2000).

Material examined. Camiguin province: Camiguin Island: Municipality of Mambajao, Barangay Bulok-bulok, 9¹5'9"N, 124²' 31 "E, 7 m a.s.l., 05-07.2017,

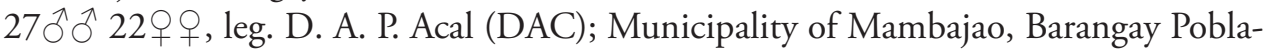

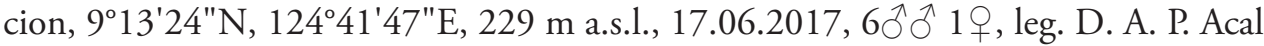

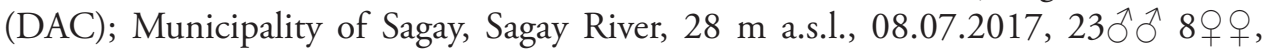
leg. D. A. P. Acal (DAC); Municipality of Catarman, Barangay Mainit, Looc River,

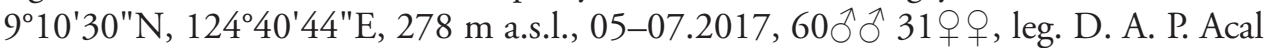
(DAC); Municipality of Catarman, Barangay Bura, 9 $9^{\circ} 10^{\prime} 4.7^{\prime \prime} \mathrm{N}, 124^{\circ} 39^{\prime} 23 " \mathrm{E}, 143 \mathrm{~m}$ a.s.l., 17.06.2017, $3 \jmath^{\Uparrow} 4$ 4우, leg. D. A. P. Acal (DAC); Tuasan Falls, Looc River, Barangay Bonbon, Municipality of Catarman, 9.176009N, 124.679768E, 11.2019, 12 exx., leg. D. A. P. Acal, R. Jaskuła (RJC); Lanao del Norte province: Barangay Mainit (between Iligan City and Cagayan de Oro), 17-20.08.1978, 10 19, leg. Cabides et Lobin (JWC); Municipality of Bacolod, Barangay Mati, $8^{\circ} 9^{\prime} 4^{\prime \prime N}, 124^{\circ} 0^{\prime} 57^{\prime E}$, $53 \mathrm{~m}$ 
a.s.l., 05-07.2017, 27ðð 17우이, leg. D. A. P. Acal (DAC); Municipality of Bacolod

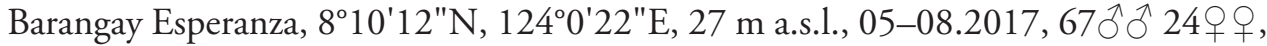
leg. D. A. P. Acal (DAC), 13.12.2018, 3ðð 1 \%, leg. R. Jaskuła (RJC); Iligan City - Ba-

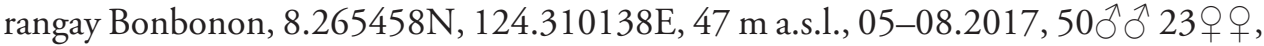
leg. D. A. P. Acal (DAC); Iligan City - Barangay Merila, 8 $12^{\prime} 17^{\prime \prime} \mathrm{N}, 1^{\circ} 4^{\circ} 15^{\prime} 24^{\prime \prime} \mathrm{E}, 18$

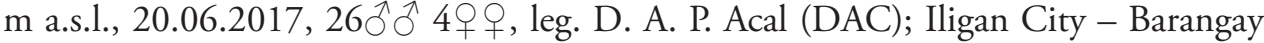

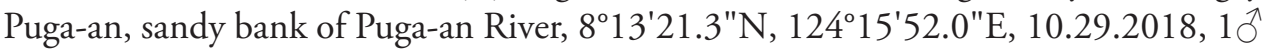
1 q, leg. C. Torres (RJC); Iligan City - Barangay Tipanoy, Tubod River, 8¹1'38.12"N, $124^{\circ} 15^{\prime} 25.38 " \mathrm{E}, 20 \mathrm{~m}$ a.s.1., 28.09.2018, $1 \mathrm{~J}^{\top}$, leg. D. A. P. Acal (DAC); Iligan City - Barangay Rogongon, Sitio Lawlawon, 8¹4'51.13N, $124^{\circ} 25^{\prime} 25.31^{\prime \prime E}, 359$ m a.s.l., 10.03.2019, 8 exx., leg. D. A. P. Acal (DAC); Misamis Occidental province: Municipality of Sinacaban, Barangay San Lorenzo, 8¹7'10"N, 12341'43"E, $800 \mathrm{~m}$ a.s.l., 06.09.2017, 5ふึ 4우, leg. D. A. P. Acal (DAC); Misamis Oriental province: Mu-

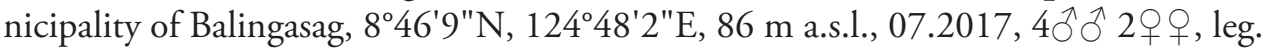
D. A. P. Acal (DAC); Municipality of Lagonglong, 8 $8^{\circ} 8^{\prime} 11^{\prime \prime} \mathrm{N}, 124^{\circ} 48^{\prime} 53^{\prime \prime E}, 90 \mathrm{~m}$

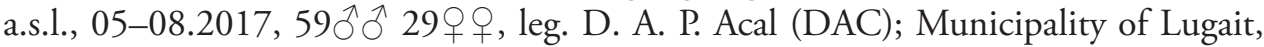
Barangay Upper Talacogon, river bank, 8²0'47.04"N, $124^{\circ} 16^{\prime} 58.80^{\prime \prime} \mathrm{E}, 11.07 .2018$, $2 \widehat{\jmath}$, leg. V. M. Mirabueno (RJC).

Habitat. The species was recorded as the most opportunistic according to habitat type among all Cicindelidae presented in this paper, found on sandy river banks, forest paths, coastal area, and unused compost pit near the river (pers. obs.).

Remarks. At least in some areas co-occur with C. lacrymosa. C. mindanaoensis was recently noted as a host of Hexathrombium (Acari: Microtrombidiidae) parasitic mites (Acal et al. - in press). This species was also observed resting on undershrub plants along the trails during rainy season.

\section{Genus Lophyra Motschulsky, 1859}

The genus Lophyra is represented in the Philippine islands by one species, known to occur also in Mindanao Island (Cabras et al. 2016a).

\section{Subgenus Spilodia Rivalier, 1961}

\section{Lophyra (Spilodia) striolata tenuiscripta (Fleutiaux, 1893)}

Figures 3G, 5E, 9D

General distribution. Subspecies known only from Indonesia and Philippines, in the second country noted only in Palawan (Cabras et al. 2016a) and Mindanao (new record).

Material examined. Bukidnon province: Mt. Kitanglad, 11-12.2014, $1 \hat{\jmath}$, leg. N. Mohagan (JWC); Lanao del Norte province: Municipality of Bacolod, Barangay Mati, 89'4"N, 1240'57"E, 53 m a.s.l., 06.2017, 26 exx., leg. D. A. P. Acal (DAC); Misamis Occidental province: Municipality of Sinacaban, Barangay San Isidro, $8^{\circ} 17^{\prime} 5^{\prime \prime} \mathrm{N}, 123^{\circ} 47^{\prime} 5^{\prime} \mathrm{E}, 269 \mathrm{~m}$ a.s.l., 05-07.2017, 78 exx., leg. D. A. P. Acal (DAC). 
Habitat. Collected along the trails of coconut field (Municipality of Bacolod, Lanao del Norte) and from cultivated corn and ginger field (Municipality of Sinacaban, Misamis Occidental) (Acal - pers. obs.).

Remarks. Lophyra striolata is a polytypic species with wide distribution in nearly the whole Oriental region (Cassola, 2000). Four subspecies currently are known from the Philippines (ssp. striolata (Illiger, 1800), spp. dorsolineolata (Chevrolat, 1845), spp. tenuiscripta (Fleutiaux, 1893), and spp. uniens (Horn, 1896)) (Cabras et al. 2016a) but at least in some cases their distribution and taxonomical status should be clarified as few subspecies were noted from the same areas.

\section{Genus Thopeutica Chaudoir, 1861}

The genus Thopeutica is the largest tiger beetle genus in the Philippines with 31 species classified in two subgenera described to date, including 27 species in subgenus Thopeutica s. str. and four species in subgenus Philippiniella (Cabras et al. 2016a, Medina et al. 2019, 2020c). Thopeutica is geographically restricted to Sulawesi and the Philippines and is one of the most exclusive genera since all except two species know from the country seem to be restricted to only one island or to very few islands (Cassola and Zettel 2006).

\section{Thopeutica (Thopeutica) angulibumerosa (Horn, 1929)}

Figure 9E

General distribution. Species endemic to the Philippines, where it was recorded from Leyte, Mindanao, and Samar; according to Cabras et al. (2016a) only general distributional data from Mindanao for this species were known, this is the first record from Northern Mindanao region.

Material examined. Bukidnon province: Barangay Kalasungay, $8^{\circ} 11^{\prime} 28^{\prime \prime} \mathrm{N}$, $125^{\circ} 5^{\prime} 54$ "E, 770 m a.s.l., 14.06.2017, 1 \%, leg. D.A.P. Acal (DAC).

Habitat. The only specimen from Northern Mindanao studied was collected in a shaded riverine area.

\section{Thopeutica (Thopeutica) darlingtonia Cassola \& Ward, 2004}

Figure $9 \mathrm{~F}$

General distribution. Species endemic to the Philippines, where it was recorded from Luzon and Mindanao; in Mindanao recorded till now only from Northern Mindanao region (Cabras et al. 2016a).

Literate data from Northern Mindanao region. Bukidnon province: Impasugong (Cabras et al. 2016a).

Habitat. No detailed data on habitat specificity in Northern Mindanao region but most probably occurring along rivers in shaded areas. 


\section{Thopeutica (Thopeutica) milanae Wiesner, 1992}

Figure 10A

General distribution. Species endemic to the Philippines, where it was recorded from Leyte, Luzon (?), Mindanao, and Samar; in Mindanao noted only from Northern Mindanao region (Cabras et al. 2016a).

Literature data from Northern Mindanao region. Bukidnon province: Impasug-ong (Cabras et al. 2016a).

Habitat. No detailed data on habitat specificity in Northern Mindanao region but most probably occurring along rivers in shaded areas.

\section{Thopeutica (Thopeutica) virginea (Schaum, 1860)}

Figure 10B

General distribution. Species endemic to Philippines where it was recorded only from Luzon (Cabras et al. 2016a) and Mindanao (new record) islands.

Material examined. Bukidnon province: Mt. Kitanglad, $11-12.2014,4$ $\widehat{~}$ 3 우, leg. N. Mohagan (JWC).

Habitat. No detailed data on habitat specificity in Northern Mindanao region but most probably occurring along rivers in shaded areas.

\section{Genus Cylindera Westwood, 1831}

The genus Cylindera is represented in the Philippine islands by 22 species, including five recorded from Mindanao Island (Cabras et al. 2016a).

\section{Subgenus Eugrapha Rivalier, 1950}

\section{Cylindera (Eugrapha) minuta (Olivier, 1790)}

Figures 2G, 3A, C-E, 5F, 10C

General distribution. Species recorded till now from Bangladesh, Brunei, Cambodia, China, India, Indonesia, Laos, Malaysia, Myanmar, Nepal, Philippines, Thailand, and Vietnam (Wiesner 1992). According to Cabras et al. (2016a) in the Philippines it is known only on the basis of general distributional data; here we present the first records of this species from Mindanao.

Material examined. Bukidnon province: Malaybalay City, Barangay Can-ayan, $8^{\circ} 11^{\prime} 31^{\prime \prime} \mathrm{N}, 125^{\circ} 9^{\prime} 13^{\prime \prime E}, 653 \mathrm{~m}$ a.s.l, 15.06.2017, 10, leg. D.A.P. Acal (DAC); Lanao del Norte province: Iligan City - Barangay Bonbonon, 8.265458N, 124.310138E, 47

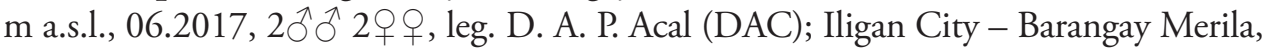
Tubod River, $8^{\circ} 12^{\prime} 17^{\prime \prime N}, 124^{\circ} 15^{\prime} 24^{\prime \prime E}, 18 \mathrm{~m}$ a.s.l., 05-07.2017, $108{ }^{\lambda} 0^{\Uparrow} 60$ 우 , leg. D. A. P. Acal (DAC), 13.12.2018, $1 \delta^{\Uparrow} 1$, leg. D. A. P. Acal (DAC); Iligan City - Barangay 

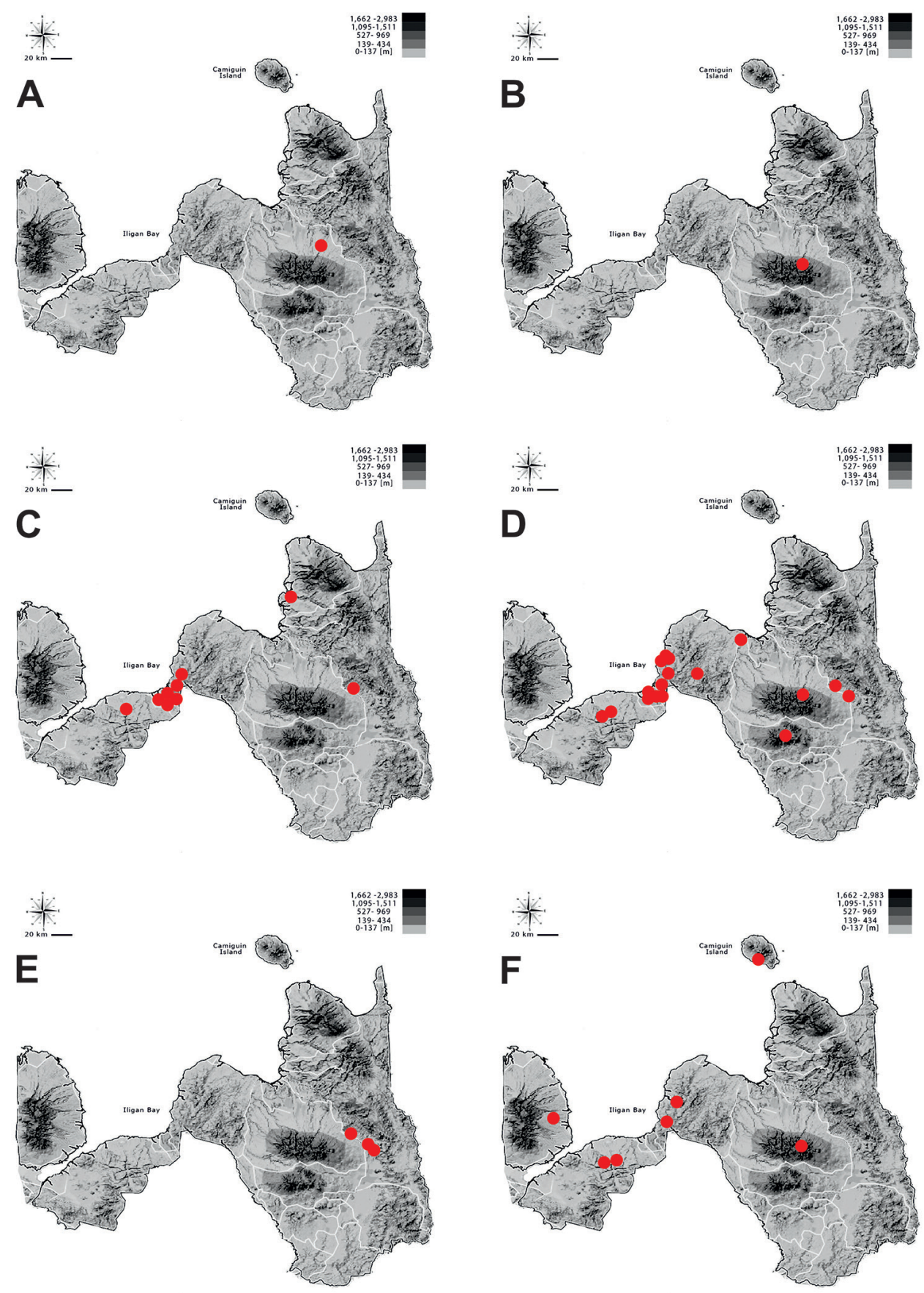

Figure 10. Distribution of A Thopeutica (T.) milanae B T. (Thopeutica) virginea C Cylindera (Eugrapha) minuta $\mathbf{D}$ C. (Ifasina) discreta elaphroides $\mathbf{E}$ C. (Ifasina) mouthiezi, and $\mathbf{F}$ C. (Ifasina) viduata in Northern Mindanao region. 
Puga-an, rocky bank of Puga-an River, $8^{\circ} 13^{\prime} 31.5^{\prime \prime N}, 1^{\circ} 4^{\circ} 16^{\prime} 08.6^{\prime \prime E}, 10.29 .2018,3{ }^{\lambda}$ $2 ㅇ$, leg. C. Torres (RJC); Iligan City - Barangay Baraas, Tubod River, 8¹2'40.23"N,

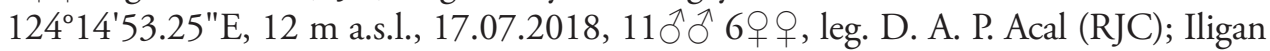
City - Barangay Tubod, Tubod River, $8^{\circ} 13^{\prime} 12.12^{\prime \prime N}, 124^{\circ} 14^{\prime} 56.00^{\prime E}$, 9 m a.s.l., 30 exx., leg. D. A. P. Acal (DAC); Municipality of Bacolod, Barangay Esperanza, 8 $10^{\prime} 12^{\prime \prime} \mathrm{N}$, $124^{\circ} 0^{\prime} 22^{\prime \prime E}, 27 \mathrm{~m}$ a.s.l., 06.2017, 10̄, leg. D. A. P. Acal (DAC); Iligan City - Barangay

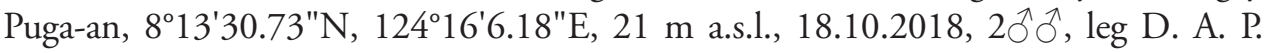
Acal (RJC); Misamis Oriental province: Municipality of Balingasag, Cabulaway River,

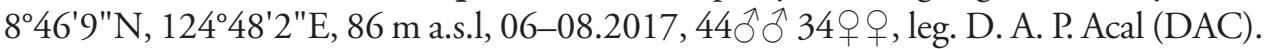

Habitat. Species occurs on sandy river banks (pers. obs.).

Remarks. This species was observed to co-occur with Calomera mindanaoensis, $C$. lacrymosa, C. angulata, and/or Cylindera discreta elaphroides (pers. obs.).

\section{Subgenus Ifasina Jeannel, 1946}

\section{Cylindera (Ifasina) discreta elaphroides (Doktouroff, 1882)}

Figures 2H, 3A, C-E, 5G-H, 10D

General distribution. Subspecies endemic to the Philippines, recorded till now from Leyte, Mindanao, Palawan, Samar (Cabras et al. 2016a), and Cebu (Cabrera et al. 2019); in Mindanao noted from Davao (Cabras et al. 2016a, b; Cabras and Wiesner 2016) and Northern Mindanao regions (new records).

Material examined. Bukidnon province: Mt. Kitanglad, 11-12.2014, 1 , leg. N. Mohagan (JWC); Malaybalay City, Barangay Kalasungay, 8¹1'28"N, 1255'54"E, $770 \mathrm{~m}$ a.s.1., 06-08.2017, 47 exx., leg. D. A. P. Acal (DAC); Malaybalay City, Barangay Can-ayan, $8^{\circ} 11^{\prime} 31^{\prime \prime} \mathrm{N}, 125^{\circ} 9^{\prime} 13^{\prime \prime E}, 653 \mathrm{~m}$ a.s.1., 06-08.2017, 226 exx., leg. D.A.P. Acal (DAC); Mt. Kalatungan, Sitio Bato, Municipality of Maramag, 11.2019., 2 exx., leg. R. Jaskuła, D. A. P. Acal (RJC); Lanao del Norte province: Iligan City - Barangay Bonbonon, 8.265458N, 124.310138E, $47 \mathrm{~m}$ a.s.1., 06.2017, 5 exx, leg. D. A. P.

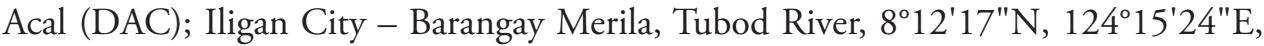
$18 \mathrm{~m}$ a.s.l., 05.2017, 3 exx., leg. D. A. P. Acal (DAC); Iligan City - Barangay Baraas, Tubod River, $8^{\circ} 12^{\prime} 40.23^{\prime \prime N}, 124^{\circ} 14^{\prime} 53.25^{\prime \prime E}, 12 \mathrm{~m}$ a.s.l., 17.07.2018, 2q q, leg. D. A. P. Acal (RJC); Iligan City - Barangay Tubod, Tubod River, 8¹3'12.12"N, $124^{\circ} 14^{\prime} 56.00^{\prime E}, 9 \mathrm{~m}$ a.s.l., 17.07.2018, 1 ex., leg. D.A.P. Acal (DAC); Iligan City - Barangay Puga-an, sandy bank of Puga-an River, 8¹3'21.3"N, 124²15'52.0"E,

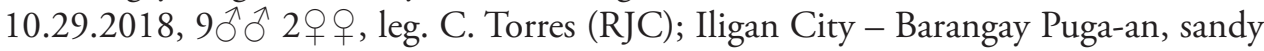
bank of Puga-an River, $8^{\circ} 13^{\prime} 29.6^{\prime \prime} \mathrm{N}, 124^{\circ} 15^{\prime} 57.8^{\prime \prime E}, 10.29 .2018$, 3 ${ }^{\lambda}{ }^{\lambda}$, leg. C. Torres (RJC); Iligan City - Barangay Tipanoy, Tubod River, $8^{\circ} 11^{\prime} 38.12^{\prime \prime} \mathrm{N}, 124^{\circ} 15^{\prime} 25.38^{\prime \prime} \mathrm{E}$, $20 \mathrm{~m}$ a.s.l., 29.08.2018, 4⿳亠ेत 1 , leg. D. A. P. Acal (RJC); Municipality of Bacolod, Barangay Esperanza, 8 $10^{\prime} 12^{\prime \prime} \mathrm{N}, 124^{\circ} 0^{\prime} 22^{\prime \prime E}, 27 \mathrm{~m}$ a.s.l., 05.2017, 1 ex., leg. D. A. P. Acal (DAC), 13.12.2018, bank of river, 2َ̧̄, leg. R. Jaskuła (RJC); Municipality

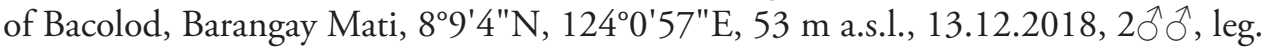
R. Jaskuła (RJC); Iligan City - Barangay Rogongon, Sitio Lawlawon, 8¹4'51.13N, 
$124^{\circ} 25^{\prime} 25.31$ "E, 359 m a.s.l., 10.03.2019, 10 exx., leg. D. A. P. Acal (DAC); Misamis Oriental province: Cagayan de Oro City, Malasag Cugman, Mapawa Nature Park,

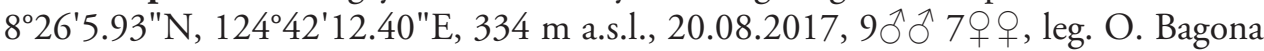
(RJC); Municipality of Lugait, Barangay Upper Talacogon, river bank, 8²0'47.04"N, $124^{\circ} 16^{\prime} 58.80^{\prime \prime E}, 08.11 .2018,2 \jmath^{\Uparrow} 2$ 우우, leg. V. M. Mirabueno (RJC), 01.12.2018,

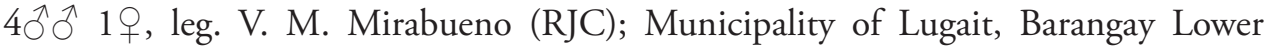
Talacogon, river bank, $8^{\circ} 20^{\prime} 47.04^{\prime \prime} \mathrm{N}, 124^{\circ} 16^{\prime} 58.80^{\prime \prime} \mathrm{E}, 03.12 .2018,20^{\top} \hat{o}^{\top} 1$ \% leg. V. M. Mirabueno (RJC); Municipality of Lugait, Barangay Aya-Aya, river bank, $8^{\circ} 20^{\prime} 00.16^{\prime \prime N}, 124^{\circ} 18^{\prime} 36.77^{\prime \prime E}, 07.11 .2018,20^{\top}{ }^{\circ}$, leg. V. M. Mirabueno (RJC).

Habitat. A riverine tiger beetles species recorded on sandy bank (per. obs.).

Remarks. Although $C$. discreta elaphroides is active mainly during sunlight hours on the river banks, it was also noted as species actively hunting during heavy rain on vertical surfaces (Cabrera et al. 2019). Some specimens were also observed resting on the leaves of Pennisetum sp. along the river (pers. obs.).

\section{Cylindera (Ifasina) mouthiezi Dheurle, 2015}

Figures $2 \mathrm{H}, 10 \mathrm{E}$

General distribution. Species endemic to Philippines (Cabras et al. 2016) where it has been recorded only from Mindanao, where it was found only from Davao and Northern Mindanao regions till now (Dheurle 2015, 2017; Cabras et al. 2016b).

Literature data from Northern Mindanao region. Bukidnon province: Cabanglasan (Dheurle 2015, 2017); Panamokan (Dheurle 2017).

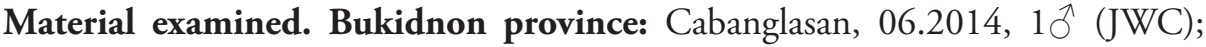
Panamokan, 06.2014, 1 ㅇ (JWC); Barangay Kalasungay, 8¹1'28"N, 1255'54"E, 770 m a.s.l., 14.06.2017, 8 exx., leg. D. A. P. Acal (DAC, RJC).

Habitat. All specimens known for us from Northern Mindanao region were collected in a shaded riverine area.

Remarks. This species co-occurs with Cylindera discreta elaphroides and Thopentica angulihumerosa.

\section{Cylindera (Ifasina) viduata (Fabricius, 1801)}

Figures 3G, 10F

General distribution. Species recorded from Bangladesh, Cambodia, China, India, Indonesia, Laos, Malaysia, Myanmar, Nepal, Papua New Guinea, Philippines, Thailand, Vietnam; in the Philippines is was noted from the following islands: Leyte, Mindanao, Palawan, and Tawi-tawi; in Mindanao recorded only on the basis of general information (Cabras et al. 2016a); here we provide the first records from Northern Mindanao region.

Material examined. Bukidnon province: Mt. Kitanglad, 11-12.2014, 1일. leg. N. Mohagan (JWC); Camiguin province: Camiguin Island, Municipality of Sagay, 
Barangay Bonbon, Sagay River, 28 m a.s.l., 08.07.2017, 2ðð, leg. D. A. P. Acal (DAC); Lanao del Norte province: Iligan City, Barangay Bonbonon, 8.265458N,

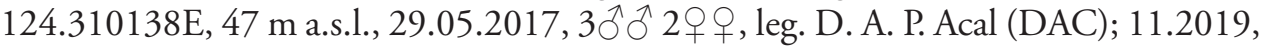
1 ex., leg. R. Jaskuła (RJC); Iligan City - Barangay Esperanza, 8¹0'12"N, $124^{\circ} 0^{\prime} 22^{\prime \prime} \mathrm{E}$, $27 \mathrm{~m}$ a.s.l., 08.06.2017, 3qq, leg. D. A. P. Acal (DAC); Municipality of Bacolod,

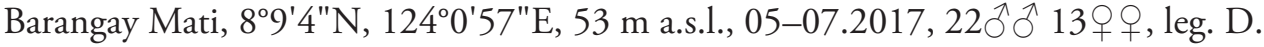
A. P. Acal (DAC), 13.12.2018, 2̧̄̋, leg. D. A. P. Acal (DAC); Misamis Occidental province: Municipality of Sinacaban, Barangay San Isidro, $8^{\circ} 17^{\prime} 5^{\prime \prime N}, 123^{\circ} 47^{\prime} 5^{\prime \prime} \mathrm{E}$, $269 \mathrm{~m}$ a.s.1., 05-07.2017, 8§へ 5우, leg. D. A. P. Acal (DAC); Misamis Oriental province: Mambuntan Falls, Barangay Lubilan, Naawan Municipality, 8.412300N, 124.351642E, 11.2019, 2exx., ad lucem, leg. R. Jaskuła (RJC).

Habitat. Species noted from trails along riverine areas, river banks, cultivated corn and ginger fields (pers. obs.).

Remarks. This species co-occurs with Lophyra striolata in agricultural fields (pers. obs.).

Provisional key to tiger beetle species known to occur in the Northern Mindanao Region

1 Metepisternum narrow, with grooves anteriorly; mesepisternum strongly elon-

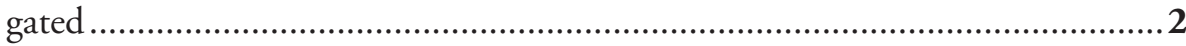

- Metepisternum relatively broad, without anterior grooves; mesepisternum usually

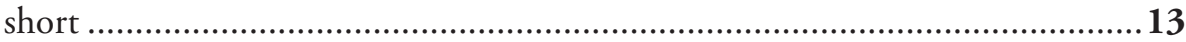

2 Outer margin of mandible without tooth; labrum 6-dentate; humeral angles of elytra and hind wings absent

3 Tribe Collyridini Brullé, 1834, Subtribe Tricondylina Naviaux, 1991

- $\quad$ Outer margin of mandible with tooth; labrum 7-dentate; humeral angles and hind wings present.

..7 Tribe Collyridini Brullé, 1834, Subtribe Collyridina Brullé, 1834

3 Base of interocular cavity at same level as neck; no distinct transverse line between neck and occiput; pronotum never both long and subrectangular; body length from 13 to $28 \mathrm{~mm}$

4 Genus Tricondyla Latreille, 1822, Subgenus Tricondyla s. str.

- Base of interocular cavity slightly higher than neck or not exactly on same extension; presence of a distinct transverse mark between neck and occiput; temples abruptly shaped from dorsal view; body length less than $17 \mathrm{~mm}$, habitus very slender

Genus Tricondyla Latreille, 1822, Subgenus Stenotricondyla Naviaux, 2002

4 Very robust species with body length 19-24 mm; elytral sculpture may be almost smooth or granular.

Tricondyla (Tricondyla) aptera punctipennis Chevrolat, 1841

- Smaller and narrower species with body length 15-20 mm; sculpture covering entire elytra (but decreasing at apex) or posterior half part almost smooth ........5 
5 Elytral sculpture deeper and covering the entire surface but decreasing at apex; median lobe of aedeagus with tip particularly acute.

Tricondyla (Tricondyla) gracilis Naviaux, 2002

- Elytral sculpture not regularly distributed with the posterior half almost smooth; median lobe of aedeagus with tip less acute.

Tricondyla (Tricondyla) elongata Horn, 1906

6 Generally smaller species (usually between 13 and $15.5 \mathrm{~mm}$ ), aedeagus not longer than $2.5 \mathrm{~mm}$........... Tricondyla (Stenotricondyla) cyanipes Eschscholtz, 1829

- Larger species, usually with body length between 14 and $17 \mathrm{~mm}$; aedeagus at least $3 \mathrm{~mm}$ long .................. Tricondyla (Stenotricondyla) cavifrons Schaum, 1862

7 Labrum very short; body smaller than $9 \mathrm{~mm}$, slender; sculpture of elytra shallow, dense and uniform

Genus Protocollyris Mandl, 1975, Protocollyris mindanaoensis (Mandl, 1974)

- Labrum longer ..................................................8 Genus Neocollyris Horn, 1901

8 Generally smaller and slender species (between 9.5 and $13.5 \mathrm{~mm}$ )

9 Genus Neocollyris Horn, 1901, Subgenus Neocollyris s. str.

- Larger and robust species with body length between 17.5 and $23 \mathrm{~mm}$.....

11 Genus Neocollyris Horn, 1901, Subgenus Heterocollyris Naviaux, 1995

9 Antennae short, reaching basal half of pronotum; color dark, not bright blue..... Neocollyris (Neocollyris) brevicula Naviaux, 1994

- Antennae longer, reaching base of pronotum; color bright blue, sometimes with

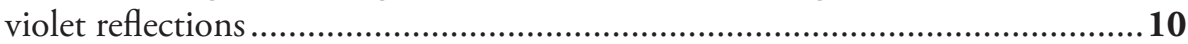

10 Vertex dilated behind eyes; pronotum short; tip of aedeagus rounded

.Neocollyris (Neocollyris) emarginata (Dejean, 1825)

- Vertex not dilated behind eyes, pronotum longer; tip of aedeagus acute.

Neocollyris (Neocollyris) albitarsis (Erichson, 1834)

11 Pronotum strongly constricted in front; aedeagus sigmoid in lateral view.....

Neocollyris (Heterocollyris) speciosa (Schaum, 1863)

- Pronotum lesser constricted in front; aedeagus not sigmoid in lateral view......12

12 Elytral sculpture dense and fine, less creased near suture

.Neocollyris (Heterocollyris) similior (Horn, 1893)

- Elytral sculpture not very coarse; creased along sutural margin.

Neocollyris (Heterocollyris) affinis (Horn, 1892)

13 Galea of maxilla reduced, one segmented; $4^{\text {th }}$ tarsal segment very shortened, with $5^{\text {th }}$ segment inserted toward the middle of its upper side; labrum long

14 Tribe Cicindelini Latreille, 1802, Subtribe Theratina Horn, 1910

- Galea of maxilla two-segmented; $4^{\text {th }}$ tarsal segment rarely shortened, $5^{\text {th }}$ segment always inserted apically; labrum often short ..................................................18

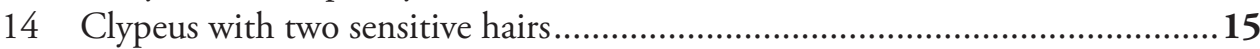

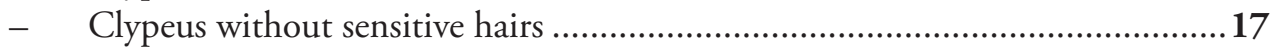

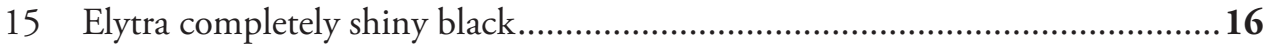

- Elytra black with brownish maculation 
16 Metasternum yellow. Therates fulvipennis everetti Erichson, 1834

- Metasternum black Therates coracinus coracinus Erichson, 1834

17 Black maculation of elytra does not reach the furrow behind the basal hump...... Therates fasciatus fasciatus (Fabricius, 1801)

- Black maculations of elytra covers the furrow behind the basal hump

Therates fasciatus pseudolatreillei Horn, 1928

18 Head, pronotum, pro- and mesosternum, base of abdomen and elytra glabrous.. 19 Tribe Cicindelini Latreille, 1802, Subtribe Prothymina Horn, 1906

- Either head, pronotum, pro- and mesosternum, base of sternum or base of elytra setose

20 Tribe Cicindelini Latreille, 1802, Subtribe Cicindelina Latreille, 1802

19 Body ventrally almost entirely glabrous except for fringe of setae on free lateral margin of hind coxae, elytra immaculate

Heptodonta nigrosericea Horn 1930 (= lumawigi Wiesner, 1980)

- Body ventrally entirely glabrous

Prothyma (Symplecthyma) heteromallicollis heteromallicollis Horn, 1909

20 Flagellum of inner sac of aedeagus coiled in a sagittal plane 20 Genus Cylindera Westwood, 1831

- Flagellum complexly coiled on both sides of the inner sac .24

21 Elytra with complete humeral lunule

Cylindera (Eugrapha) minuta (Olivier, 1790)

- Humeral lunule absent, himeral maculations, if present, split in two dots 22 Genus Cylindera Westwood, 1831, Subgenus Ifasina Jeannel, 1946

22 Elytra without any humeral maculations

Cylindera (Ifasina) viduata (Fabricius, 1801)

- Humeral maculations present.

23 Humeral maculations consists of a small posthumeral dot only, humeral dot absent .................. Cylindera (Ifasina) discreta elaphroides (Doktouroff, 1882)

- Humeral maculations constist of a large humeral and a large posthumeral dot.... Cylindera (Ifasina) mouthiezi Dheurle, 2015

24 Flagellum of inner sac of aedeagus with more than four windings....

25 Genus Thopeutica Schaum, 1861

- Flagellum with less than four windings ..... .28

25 Prothorax mostly glabrous, setation restricted to pleurosternal suture or along the anterior margin

- Pronotum with lateral margins and/or lateral angles variably setose.

26 Labrum with 10-14 submarginal setae; females without elytral mirror Thopeutica (Thopeutica) virginea (Schaum, 1860)

- Labrum with 8 submarginal and mesal setae; females with elytral mirror spot..... Thopeutica (Thopeutica) milanae Wiesner, 1992

27 Elytra with humeral callus; prosternum distinctly longer than wide Thopeutica (Thopeutica) angulihumerosa (Horn, 1929)

- Elytra without humeral callus; prosternum slightly longer than wide Thopeutica (Thopeutica) darlingtonia Cassola et Ward, 2004 
28 Labrum with more than ten marginal setae

29 Genus Calomera Motschulsky, 1862

- Labrum with four to eight marginal setae. Genus Lophyra Motschulsky, 1859, Lophyra (Spilodia) striolata tenuiscripta (Fleutiaux, 1893)

29 Elytral maculation consists of humeral and apical lunule marginal band and middle band, which all are more or less connected with one another; female elytra expanded laterally Calomera angulata angulata (Fabricius, 1798)

- Elytral maculation consists of apical lunule and five or six dots; female elytra are not expanded laterally

30 Elytra with apical lunule and five dots (humeral, subhumeral, submarginal, discal and another submarginal one below the other submarginal dot); elytra velvety black, without visible punctuation throughout Calomera cabigasi Cassola, 2011

- Elytra with apical lunule and six dots (as mentioned above, plus an upper discal dot); elytra with visible punctuation 31

31 Elytra greenish or bluish, with blue green punctuation; aedeagus short, with a tiny hook shaped tip Calomera lacrymosa (Dejean,1825)

- Elytra darker, nearly velvety black, punctuation nearly not visible in apical half of elytra; aedeagus longer, ending in a long straight apical beak

Calomera mindanaoensis (Cassola, 2000)

\section{Discussion}

Tiger beetle fauna of Northern Mindanao vs. fauna of the entire island and country

Thirty species (including two with two subspecies each) classified in ten genera are actually known from Northern Mindanao region (Table 2, Figures 5-9), which constitute $56 \%$ of Cicindelidae fauna of Mindanao and $21 \%$ of the Philippines. Three of these species are known as endemics of Mindanao Island (Neocollyris similior, Calomera cabigasi, and Cylindera (Ifasina) mouthiezi), one more (Calomera mindanaoesis) is restricted only to Mindanao and Camiguin islands, while 22 taxa occur only in the Philippines (Cabras et al. 2016a). Among the recorded taxa, Neocollyris speciosa, Calomera angulata, Cylindera minuta, and Lophyra striolata tenuiscripta, Thopeutica virginea were noted for the first time from Mindanao island, and moreover, an additional seven species had not been reported from Northern Mindanao region before (Tricondyla cavifrons, Neocollyris similior, Prothyma heteromallicollis heteromallicollis, Thopeutica angulihumerosa, Cylindera discreta elaphroides, C. mouthiezi, C. viduata). The highest number of taxa was noted from Bukidnon (24 species, $80 \%$ of Northern Mindanao fauna) Misamis Oriental (15 species, $50 \%$ of fauna), and Lanao del Norte provinces (14 species, $47 \%$ of fauna). Misamis Occidental and Camiguin provinces were characterized by $23 \%$ and $7 \%$ of fauna respectively (Table 2). The number of recorded Cicindelidae taxa seems to depend on the surface area of the province, as Bukidnon is the largest 
Table 2. Distribution of tiger beetles in administrative provinces of Northern Mindanao region.

\begin{tabular}{|c|c|c|c|c|c|}
\hline \multirow[t]{2}{*}{ Species } & \multicolumn{5}{|c|}{ Provinces of Northern Mindanao region } \\
\hline & Bukidnon & Camiguin & $\begin{array}{l}\text { Lanao } \\
\text { del Norte }\end{array}$ & $\begin{array}{c}\text { Misamis } \\
\text { Occidental }\end{array}$ & $\begin{array}{l}\text { Missamis } \\
\text { Oriental }\end{array}$ \\
\hline Tricondyla (Tricondyla) aptera punctipennis Chevrolat, 1841 & + & & + & & \\
\hline Tricondyla (Tricondyla) elongata Horn, 1906 & + & & + & + & + \\
\hline Tricondyla (Tricondyla) gracilis Naviaux, 2002 & & & & & + \\
\hline Tricondyla (Stenotricondyla) cyanipes Eschscholtz, 1829 & & & & & + \\
\hline Tricondyla (Stenotricondyla) cavifrons Schaum, 1862 & + & & + & & \\
\hline Neocollyris (Neocollyris) albitarsis (Erichson, 1834) & + & & & & \\
\hline Neocollyris (Neocollyris) brevicula Naviaux, 1994 & & & + & & \\
\hline Neocollyris (Neocollyris) emarginata (Dejean, 1825) & + & & & & \\
\hline Neocollyris (Heterocollyris) affinis (Horn, 1892) & + & & & & + \\
\hline Neocollyris (Heterocollyris) similior (Horn, 1893) & & & + & & + \\
\hline Neocollyris (Heterocollyris) speciosa (Schaum, 1863) & + & & & & \\
\hline Protocollyris mindanaoensis (Mandl, 1974) & & & + & & \\
\hline Therates coracinus coracinus Erichson, 1834 & + & & + & & + \\
\hline Therates fasciatus fasciatus (Fabricius, 1801) & + & + & + & & + \\
\hline Therates fasciatus pseudolatreillei Horn, 1928 & + & & + & + & + \\
\hline Therates fulvipennis bidentatus Chaudoir, 1861 & + & & + & & \\
\hline Therates fulvipennis everetti Erichson, 1834 & + & & & & \\
\hline Prothyma (Symplecthyma) heteromallicollis heteromallicollis Horn, 1909 & + & & + & & + \\
\hline Heptodonta nigrosericea (W. Horn, 1930) & + & & & + & + \\
\hline Calomera angulata angulata (Fabricius, 1798) & & & + & & \\
\hline Calomera cabigasi Cassola, 2011 & + & & & & + \\
\hline Calomera lacrymosa (Dejean,1825) & & & + & + & + \\
\hline Calomera mindanaoensis (Cassola, 2000) & + & + & + & + & + \\
\hline Lophyra (Spilodia) striolata tenuiscripta (Fleutiaux, 1893) & + & & + & + & \\
\hline Thopeutica (Thopeutica) angulibumerosa (Horn, 1929) & + & & & & \\
\hline Thopeutica (Thopeutica) darlingtonia Cassola et Ward, 2004 & + & & & & \\
\hline Thopeutica (Thopeutica) milanae Wiesner, 1992 & + & & & & \\
\hline Thopeutica (Thopeutica) virginea (Schaum, 1860) & + & & & & \\
\hline Cylindera (Eugrapha) minuta (Olivier, 1790) & + & & + & & + \\
\hline Cylindera (Ifasina) discreta elaphroides (Doktouroff, 1882) & + & & + & & + \\
\hline Cylindera (Ifasina) mouthiezi Dheurle, 2015 & + & & & & \\
\hline Cylindera (Ifasina) viduata (Fabricius, 1801) & + & + & + & + & + \\
\hline Total & 25 & 3 & 18 & 7 & 16 \\
\hline
\end{tabular}

one and Camiguin is the smallest area. Since not all types of habitats were studied in particular provinces, additional tiger beetle species inhabiting in Northern Mindanao region are expected to be found with more extensive fieldwork in the future.

\section{Tiger beetles and their habitats}

Among Cicindelidae taxa recorded in Northern Mindanao region both epigeic ( $\mathrm{Cal}$ omera, Cylindera, Heptodonta, Lophyra, Prothyma, Thopeutica) and arboreal (Therates, Neocollyris, Protocollyris, Tricondyla) species were noted. Most of the epigeic species are recognized as riverine tiger beetles (all Calomera and most Cylindera except $C$. viduata, as well as Heptodonta nigrosericea, Lophyra, Thopeutica) occurring on sandy and sunny banks or on shaded banks of medium and large rivers. Among the epigeic Cicindelidae only Cylindera viduata, Prothyma heteromallicollis heteromallicollis, and 
some Lophyra striolata tenuiscripta were noted as forest beetles occupying different sandy areas. Our data from Northern Mindanao region confirm observations both from other regions of Mindanao (Cabras et al. 2016b; Cabras and Wiesner 2016; Medina 2020; Medina et al. 2020; Pepito et al. 2020) and different parts of the world including e.g., some regions of North America (Pearson et al. 1997), Africa (Jaskuła 2015; Jaskuła et al. 2015; Jaskuła and Rewicz 2015; Jaskuła and Płociennik 2020), Asia (Dangalle et al. 2014) or Europe (Jaskuła 2011; Jaskuła et al. 2019), as tiger beetles are known to prefer riverine habitats not only because of adequate water and food resources but also for protection from predators and human disturbances (Bhargav and Uniyal 2008). In many regions of the world the highest diversity and species richness of epigeic tiger beetles are noted mostly on lowland areas that had a variety of habitats such as coastal areas, river banks, grasslands, and sand dunes attractive for tiger beetles (e.g., Pearson and Cassola 1992; Pearson et al. 1997; Jaskuła 2011, 2015; Dangalle et al. 2014; Jaskuła et al. 2019; Jaskuła and Płociennik 2020). On the other hand, in the tropical regions large number of Cicindelidae are typical arboreal taxa (e.g., Wiesner 1992; Pearson and Vogler 2001; Moravec 2007; Dangalle 2018) and large forests, especially natural ones, are characterized by high species diversity of such tiger beetles. In Northern Mindanao region, where more than $60 \%$ of its entire area is classified as forest land, $48 \%$ of all recorded Cicindelidae taxa are noted as arboreal taxa (Table 2). This number is expected to increase in the future as several additional arboreal species are known from other parts of Mindanao, including areas located close to the administrative border of Northern Mindanao region (Cabras et al. 2016a). Moreover, in case of some species, only general distributional data from Mindanao are known (e.g., Protocollyris okajimai Mandl, 1982, Neocollyris rugei Horn, (1892) N. erichsoni (Horn, 1892), N. chaudoiri (Horn, 1892) (Naviaux 1994)), it cannot be excluded that some of these taxa were collected (and actually inhabit) in the Northern Mindanao region. On the other hand it is necessary to note that many areas in Mindanao, including Northern Mindanao region, are under large impacts of human activities, and as a result many tiger beetle habitats are regularly destroyed. Forest destruction, including deforestation in all sorts and forms such as illegal logging, mining, agricultural expansion, quarrying, over-extraction of plant biota for fuel and other domestic uses, or conversion of land into human settlement are among the rampant problems in the area (Magdalena 1996; Carandang et al. 2012).

\section{Conclusions}

Present data on diversity and distribution of Cicindelidae of Northern Mindanao region clearly suggest that the area (especially riverine habitats and forests) is unique for tiger beetle fauna which includes a significant number of both species endemic to 
Mindanao and to the entire country. Moreover, the lack of data on Cicindelidae in many areas in Mindanao Island and in the country is evident, and for many species, only single records are known. As the region is characterized by a large mosaic of still poorly explored habitats (e.g., forests in the mountains, upper parts of river systems), and more than $12 \%$ of all species noted from Mindanao were discovered and described as new for science only during last two decades, it should be expected that future studies will provide many new and important distributional data and probably will describe new Cicindelidae taxa.

\section{Acknowledgements}

We would like to thank Obed Bagona, Annaly B. Lapore, Vera Marie Mirabueno, Charity Torres, and Łukasz Trębicki who made possible to study material of Cicindelidae collected by them; Obed Bagona and Annaly B. Lapore also provided photographs of tiger beetle habitats. The first author would like to thank to the following persons for their kind help during MSc fieldwork: Penny Acal, Kieth Jay Acal, Angel Luz M. Lesondra. The following persons helped DAPA and RJ to collect insect material during the Agad-Agad and Naawan trips: Noville Jay Ebina, Christine Jean Abapo, Gypsie Claudine Café, Norjanah Campong, Marco Luis Lumontod, Grapesy Violet, Angeleth Taotao, Jamalia Macatanong, Fatimah Radiamodah, Sitty Adna Camama, Jonaisah Abdullah, Nurhana Sabaani, Eddie Modejar, Radonna Jessah Christy Maandig, and during the Bukidnon and Camiguin trips: Elbert B. Caballero, Philip Noel Banaag, Rico Ray T. Mendoza. Finally, we would like to thank to our guides and cooks in Bukidnon Province, Mt. Kalatungan: Jose A. Sabares, Joenel A. Sabares, Nelia A. Sabares; Mt. Kitanglad: Don Espiñosa, Bong Espiñosa, Thelma Aatoque; and in Camiguin Island: Gloria D. Abian, Johner C. Abian, Hilda Lsserna. Permissions to conduct field collections were obtained from the Department of Environmental and Natural Resources (DENR-Region 10) through a Wildlife Gratuitous Permits no. R10 2017-23, no. R10 2019-81, and Wildlife Export permit no. R10-2018-02, Wildlife Gratuitous Permit no. R10 2019-48 (Mt. Kalatungan Range Natural Park), Wildlife Gratuitous Permit no. R10 2019-89 (Camiguin Island), Wildlife Gratuitous Permit no. R10 2019-88 (Bukidnon, Mt. Kitangland Range National Park). The Department of Science and Technology - Accelerated Science and Technology Human Resource Development Program (DOST-ASTHRDP) is gratefully acknowledged by DAPA for all financial support during the collection of materials and the fellowship through the Research Enrichment Program (Sandwich Program). Two visits in the Philippines of RJ were possible due to financial support by the Faculty of Biology and Environmental Protection, University of Lodz, Poland. Finally, we would like to thank Thorsten Assmann (Germany), Andrey Matalin (Russia), and Rikjan Vermeulen (The Netherlands) for their valuable comments to the first version of the manuscript. 


\section{References}

Acal DAP, Nuñeza OM, Saboori A, Zawal A, Jaskuła R (in press) Not always alone: Philippine Calomera tiger beetles (Coleoptera: Cicindeldiae) and their ectoparasitic mites. International Journal for Parasitology: Parasites and Wildlife.

Anichtchenko A, Medina MN (2019) A new Neocollyris (Heterocollyris) subspecies from Mindanao, Philippines (Coleoptera, Carabidae, Cicindelinae). Acta Biologica Universitatis Daugavpiliensis 19(1): 13-15.

Anitchenko A, Medina MN (2020) Contribution to the knowledge of three species of the genus Thopeutica Schaum, 1861 from the Philippines (Coleoptera: Carabidae: Cicindelinae). Zootaxa 4748(2): 389-395. https://doi.org/10.11646/zootaxa.4748.2.11

Bhargav VK, Uniyal VP (2008) Communal roosting of tiger beetles (Cicindelidae: Coleoptera) in the Shivalik Hills, Himachal Pradesh, India. Cicindela 40(1-2): 1-12.

Bouquet Y (2017) The Philippine Archipelago. Springer Geography. Springer International Publishing, 848 pp. https://doi.org/10.1007/978-3-319-51926-5

Cabras AA, Cabigas E, Wiesner J (2016a) Updated checklist of tiger beetles in the Philippines (Coleoptera, Carabidae, Cicindelidae) $\left(131^{\text {th }}\right.$. Contribution towards the knowledge of Cicindelidae). Lambillionea 116(3): 188-201.

Cabras AA, Medina MND, Wiesner J (2016b) Tiger beetles (Coleoptera: Carabidae: Cicindelinae) of Compostela Valley Province, Mindanao Island, Philippines. Biotropia 23(2): 137-143. https://doi.org/10.11598/btb.2016.23.2.564

Cabras AA, Wiesner J (2016) Tiger beetles (Coleoptera: Carabidae: Cicindelinae) of Mainit Hotspring and Mati Protected Landscape, Mindanao with notes on their ecology and threats. International Research Journal of Biological Sciences 5(9): 1-6.

Cabrera G, Nuñeza OM, Wiesner J, Jaskuła R (2019) Hunting in the rain: unusual behaviour in tiger beetle Cylindera discreta elaphroides in tropical forest in Cebu Island, Philippines (Coleoptera: Cicindelidae). The Coleopterologists Bulletin 73(2): 408-410. https://doi. org/10.1649/0010-065X-73.2.408

Carandang AP, Bugayong LA, Dolom PC, Garcia LN, Villanueva MMB (2012) Analysis of Key Drivers of Deforestation and Forest Degradation in the Philippines. Deutsche Gesellschaft für Internationale Zusammenarbeit (GIZ) GmbH.

Cassola F (2000) Studies on tiger beetles. CII. The Cicindelidae collected by Roland A. Müller in the Philippine Islands, with description of three new species (Coleoptera: Cicindelidae). Zoologische Mededelingen 73(33): 491-509. https://doi.org/10.1016/S0006-3207(00)00034-3

Cassola F (2011) Studies of tiger beetles. CLXXXIX. A new Calomera species from Mindanao, Philippines (Coleoptera, Cicindelidae). Spixiana 34(1): 129-131.

Cassola F, Pearson DL (2000) Global patterns of tiger beetle species richness (Coleoptera: Cicindelidae): their use in conservation planning. Biological Conservation 95: 197-208.

Cassola F, Ward RD (2004) Systematics and zoogeography of the Philippine species of the genus Thopeutica Chaudoir, 1861. Annali del di Storia Naturale di Genova 96: 1-32.

Cassola F, Zettel H (2006) A new species and a new record of Thopeutica Chaudoir, 1861 from Polillo Island, Quezon Province, the Philippines (Coleoptera: Cicindelidae). Zeitschrift der Arbeitsgemeinschaft der Österreichischen Entomologen 58: 45-52. 
Dangalle CD (2018) The forgotten tigers: the arboreal tiger beetles of Sri Lanka. Journal of National Science Foundation of Sri Lanka 46(3): 241-252. https://doi.org/10.4038/jnsfsr. v46i3.8477

Dangalle CD, Pallewatta N, Vogler AP (2014) Distribution and habitat preferences of tiger beetles (Coleoptera: Cicindelidae) of the riverine ecosystems of Sri Lanka. Journal of Threatened Taxa 6(9): 6195-6203. https://doi.org/10.11609/JoTT.o3674.6195-203

Dejarme-Calalang GM, Colinet G (2014) A review of soils and crops in the Bukidnon Highlands of Northern Mindanao, the Philippines. Biotechnology, Agronomy, Society and Environment 18(4): 544-557.

Dheurle C (2015) Cylindera (Ifasina) mouthiezi, nouvelle espèce des Philippines (Coleoptera Cicindelidae). L'Entomologiste 71(2): 117-118.

Dheurle C (2016) Une nouvelle espèce de Cylindera Westwood, 1831 des Philippines (Coleoptera, Cicindelidae). Bulletin de la Société Entomologique de France 121(1): 37-40.

Dheurle C (2017) Description de Ia femelle de Cylindera (Ifasina) mouthiezi Dheurle, 2015 (Coleoptera, Cicindelidae). Le Coléoptériste 20(3): 187-188.

Dheurle C (2019) Une nouvelle espèce du genre Protocollyris Mandl, 1975 des Philippines (Coleoptera, Cicindelidae, Collyrinae). Le Coléoptériste 22(2): 79-80.

Duran DP, Gough HM (2020) Validation of tiger beetles as distinct family (Coleoptera: Cicindelidae), review and reclassification of tribal relationships. Systematic Entomology 45: 723-729. https://doi.org/10.1111/syen.12440

Görn S (2020) Revision of the Oriental tiger beetle genus Heptodonta Hope, 1838 (Coleoptera: Cicindelidae). Zootaxa 4875(1): 001-062. https://doi.org/10.11646/zootaxa.4875.1.1

Jaskuła R (2011) How unique is the tiger beetle fauna (Coleoptera. Cicindelidae) of the Balkan Peninsula? ZooKeys 100: 487-502. https://doi.org/10.3897/zookeys.100.1542

Jaskuła R (2015) The Maghreb - one more important biodiversity hot spot for tiger beetle fauna in the Mediterranean region. ZooKeys 482: 35-53. https://doi.org/10.3897/zookeys.482.8831

Jaskuła R, Płóciennik M (2020) Water is needed to exist: habitat preferences of tiger beetles (Coleoptera: Cicindelidae) in a desert country. Insects 11: e809. https://doi.org/10.3390/ insects11110809

Jaskuła R, Płóciennik M, Schwerk A (2019) From a climate zone to a microhabitat - environmental factors affecting coastal distribution of tiger beetles (Coleoptera: Cicindelidae) in the south-eastern European biodiversity hotspot. PeerJ 7: e6676. https://doi.org/10.7717/ peerj.6676

Jaskuła R, Rewicz T (2015) Tiger beetles (Coleoptera: Carabidae: Cicindelinae) of Tunisia: distribution, phenology, taxa list and new records. African Entomology 23(2): 467-485. https://doi.org/10.4001/003.023.0217

Jaskuła R, Rewicz T, Kwiatkowski K (2015) Tiger beetle fauna (Coleoptera: Carabidae, Cicindelinae) of Morocco: distribution, phenology and list of taxa. Entomologica Fennica 26: 132-155. https://doi.org/10.33338/ef.52825

Lasco RD, Pulhin FB, Sanchez PA, Villamor GB, Villegas KA (2008) Climate change and forest ecosystems in the Philippines: vulnerability, adaptation and mitigation. Journal of Environmental Science and Management 11(1): 1-14. 
López-López A, Vogler AP (2017) The mitogenome phylogeny of Adephaga (Coleoptera). Molecular Phylogenetics and Evolution 114: 166-174. https://doi.org/10.1016/j. ympev.2017.06.009

Magdalena FV (1995) Population growth and the changing ecosystem in Mindanao. Journal of Social Issues in Southeastern Asia 11(1): 105-127. https://doi.org/10.1355/SJ11-1E

Marohomsalic JS, Nuñeza OM, Michalski M, Wiesner J, Jaskuła R (2021) Alien and native tree species having extrafloral nectaries as favourite hunting area for arboreal endemic Philippine tiger beetles (Coleoptera: Cicindelidae) in human disturbed habitat in Lanao del Sur Province, Mindanao, Philippines. Ecology and Evolution 00: 1-7. https://doi. org/10.1002/ece3.7149

Medina MND (2020) Notes on habitat specificity and occurrence of some Philippine tiger beetles: Input to tropical biodiversity conservation. Journal of Tropical Coleopterology 1(2): 25-34.

Medina MND, Cabras AA, Wiesner J (2019) Thopeutica petertaylori, a new tiger beetle species (Coleoptera: Cicindelidae) from Mindanao, Philippines. Insecta Mundi 0733: 1-5.

Medina MND, Cabras AA, Ibanez J, Opiso G, Villanueva RJT (2020a) Annotated list of tiger beetles (Coleoptera: Cicindelidae) in Calanasan, Apayao Province, Luzon, Philippines. Checklist 16(1): 37-45. https://doi.org/10.15560/16.1.37

Medina MND, Cabras AA, Ramillano H, Villanueva RJT (2020b) Tiger beetles (Coleoptera: Cicindelinae) of Davao Region, Mindanao, Philippines. Journal of Threatened Taxa 12(4): 15460-15467. https://doi.org/10.11609/jott.5102.12.4.15460-15467

Medina MND, Cabras AA, Villanueva RJT (2020c) Thopeutica (Thopeutica) barsevskisi sp. n. a new tiger beetle (Coleoptera: Cicindelidae) in Bohol Island Philippines. Baltic Journal of Coleopterology 20(1): 95-100.

Moravec J (2007) Tiger beetles of Madagascar (Vol. 1). A monograph of the genus Pogonostoma (Coleoptera: Cicindelidae). Nakladatelstvi-Kabourek, Zlín, 499 pp.

Navarrete I, Peque D, Macabuhay M (2018) Soil information as a reforestation decisionmaking tool and its implication for forest management in the Philippines. In: Lopez M, Suryomenggolo J (Eds) Environmental Resources Use and Challenges in Contemporary Southeast Asia. Asia in Transition (Vol. 7). Springer, Singapore, 97-116. https://doi. org/10.1007/978-981-10-8881-0_5

Naviaux R (1994) Les Collyris (Coleoptera, Cicindelidae). Revision des genres et description de nouveaux taxons. Bulletin mensuel de la Société linnéenne de Lyon 63(4): 1-332. https:// doi.org/10.3406/linly.1994.11023

Naviaux R (2002) Tricondylina (Coleoptera, Cicindelidae). Mémoires de la Société Entomologique de France 5: 1-106.

Pearson DL, Barraclough TG, Vogler AP (1997) Distributional maps for North American species of tiger beetles (Coleoptera: Cicindelidae). Cicindela 29: 33-84.

Pearson DL, Cassola F (1992) World-wide species richness patterns of tiger beetles (Coleoptera: Cicindelidae): indicator taxon for biodiversity and conservation studies. Conservation Biology 6: 376-391. https://doi.org/10.1046/j.1523-1739.1992.06030376.x

Pearson DL, Vogler AP (2001) Tiger Beetles: the Evolution, Ecology and Diversity of the Cicindelids. Cornell University Press, Ithaca/London, 333 pp. 
Pepito J, Torrejos C, Cabras A, Medina MN, Cudera R (2020) Preliminary List of Carabidae and Cicindelidae (Coleoptera) Fauna in Lake Holon, T'boli, South Cotabato, Philippines. Journal of Tropical Coleopterology 1(2): 35-46.

Trautner J, Schawaller W (1996) Larval morphology, biology and faunistics of Cicindelidae (Coleoptera) from Leyte, Philippines. Tropical Zoology 9(1): 47-59. https://doi.org/10.1 080/03946975.1996.10539302

Wiesner J (1992) Verzeichnis der Sandlaufkäfer der Welt - Checklist of the Tiger Beetles of the World. Verlag Erna Bauer, Keltern, Germany, 364 pp.

Wiesner J (2020) Checklist of the Tiger Beetles of the World ( $2^{\text {nd }}$ Edition). Winterwork, Borsdorf, $540 \mathrm{pp}$.

Zettel H, Pangantihon CV (2017) Two new tiger beetle species of the Therates fasciatus group (Coleoptera: Carabidae: Cicindelinae). Zeitschrift der Arbeitsgemeinschaft Österreichischer Entomologen 69: 95-104.

Zettel H, Wiesner J (2018) Cylindera (Conidera) mindoroana sp. n. (Coleoptera: Cicindelidae), a new tiger beetle species from the Philippines. Insecta Mundi 0632: 1-10. 